\title{
DISPERSIVE ESTIMATES FOR HIGHER DIMENSIONAL SCHRÖDINGER OPERATORS WITH THRESHOLD EIGENVALUES I: THE ODD DIMENSIONAL CASE
}

\author{
MICHAEL GOLDBERG AND WILLIAM R. GREEN
}

\begin{abstract}
We investigate $L^{1}\left(\mathbb{R}^{n}\right) \rightarrow L^{\infty}\left(\mathbb{R}^{n}\right)$ dispersive estimates for the Schrödinger operator $H=-\Delta+V$ when there is an eigenvalue at zero energy and $n \geq 5$ is odd. In particular, we show that if there is an eigenvalue at zero energy then there is a time dependent, rank one operator $F_{t}$ satisfying $\left\|F_{t_{t}}\right\|_{L^{1} \rightarrow L^{\infty}} \lesssim|t|^{2-\frac{n}{2}}$ for $|t|>1$ such that

$$
\left\|e^{i t H} P_{a c}-F_{t}\right\|_{L^{1} \rightarrow L^{\infty}} \lesssim|t|^{1-\frac{n}{2}}, \quad \text { for }|t|>1 .
$$
\end{abstract}

With stronger decay conditions on the potential it is possible to generate an operatorvalued expansion for the evolution, taking the form

$$
e^{i t H} P_{a c}(H)=|t|^{2-\frac{n}{2}} A_{-2}+|t|^{1-\frac{n}{2}} A_{-1}+|t|^{-\frac{n}{2}} A_{0},
$$

with $A_{-2}$ and $A_{-1}$ finite rank operators mapping $L^{1}\left(\mathbb{R}^{n}\right)$ to $L^{\infty}\left(\mathbb{R}^{n}\right)$ while $A_{0}$ maps weighted $L^{1}$ spaces to weighted $L^{\infty}$ spaces. The leading order terms $A_{-2}$ and $A_{-1}$ vanish when certain orthogonality conditions between the potential $V$ and the zero energy eigenfunctions are satisfied. We show that under the same orthogonality conditions, the remaining $|t|^{-\frac{n}{2}} A_{0}$ term also exists as a map from $L^{1}\left(\mathbb{R}^{n}\right)$ to $L^{\infty}\left(\mathbb{R}^{n}\right)$, hence $e^{i t H} P_{a c}(H)$ satisfies the same dispersive bounds as the free evolution despite the eigenvalue at zero.

\section{INTRODUCTION}

The free Schrödinger evolution on $\mathbb{R}^{n}$,

$$
e^{-i t \Delta} f(x)=\frac{1}{(4 \pi i t)^{\frac{n}{2}}} \int_{\mathbb{R}^{n}} e^{-i|x-y|^{2} / 4 t} f(y) d y
$$

maps $L^{1}\left(\mathbb{R}^{n}\right)$ to $L^{\infty}\left(\mathbb{R}^{n}\right)$ with norm bounded by $|4 \pi t|^{-\frac{n}{2}}$. As an immediate consequence, solutions whose initial data belong to $L^{1}\left(\mathbb{R}^{n}\right) \cap L^{2}\left(\mathbb{R}^{n}\right)$ experience time decay with respect to the supremum norm even while a conservation law holds the $L^{2}$ norm constant. While both the dispersive bound and the conservation law can be verified with elementary Fourier

Date: October 20, 2018.

This work was partially supported by a grant from the Simons Foundation (Grant Number 281057 to the first author.) The second author acknowledges the support of an AMS Simons Travel grant and a Rose-Hulman summer professional development grant. 
analysis, they act in concert to imply Strichartz estimates for the free Schrödinger equation, which are not readily apparent in either the physical-space or frequency-space description of the propagator.

The stability of dispersive estimates under pertubration by a short range potential, that is for a Schrödinger operator of the form $H=-\Delta+V$, where $V$ is real-valued and decays at spatial infinity, is a well-studied problem. Where possible, the estimate is presented in the form

$$
\left\|e^{i t H} P_{a c}(H)\right\|_{L^{1}\left(\mathbb{R}^{n}\right) \rightarrow L^{\infty}\left(\mathbb{R}^{n}\right)} \lesssim|t|^{-n / 2}
$$

Projection onto the continuous spectrum of the spectrum of $H$ is needed as the perturbed Schrödinger operator may possess pure point spectrum that experiences no decay at large times.

The first results in this direction [23, 18, 16, 22, 17] studied mappings between weighted $L^{2}\left(\mathbb{R}^{n}\right)$ in place of $L^{1}\left(\mathbb{R}^{n}\right)$ and $L^{\infty}\left(\mathbb{R}^{n}\right)$. Estimates of type (1) are proved in [21, 28, 25, 13, [26, 4, 6, 3, 15] in various dimensions, and with different characterizations of the potential $V(x)$. For a more detailed history, see the survey paper [27].

If the potential satisfies a pointwise bound $|V(x)| \lesssim(1+|x|)^{-\beta}$ for some $\beta>1$, then the spectrum of $H$ is purely absolutely continuous on $(0, \infty)$, see [24, Theorem XIII.58]. This leaves two principal areas of concern: a high-energy region when the spectral parameter $\lambda$ satisfies $\lambda>\lambda_{1}>0$ and a low-energy region $0<\lambda<\lambda_{1}$ for some fixed constant $\lambda_{1}>0$.

It was observed by the first author and Visan [14] in dimensions $n \geq 4$, that it is possible for the dispersive estimate to fail as $t \rightarrow 0$ if the potential is not sufficiently differentiable even for a bounded compactly supported potential. The failure of the dispersive estimate is a high energy phenomenon. Positive results have been obtained in dimensions $n=4,5$ by Cardoso, Cuevas, and Vodev [4 using semi-classical techniques assuming that $V$ has $\frac{n-3}{2}+\epsilon$ derivatives, and by Erdoğan and the second author in dimensions $n=5,7,[6]$ under the assumption that $V$ is differentiable up to order $\frac{n-3}{2}$, which is the optimal smoothness requirement in light of the counterexample in [14. The decay assumptions on the potential in [6] were later relaxed by the second author in [15]. The much earlier result of Journé, Soffer, Sogge 21] requires that $\widehat{V} \in L^{1}\left(\mathbb{R}^{n}\right)$ in lieu of a specific number of derivatives.

In dimensions $n \leq 4$ in addition to zero energy eigenvalues, there is another class of obstructions at zero energy called resonances. Resonances are distributional solutions of $H \psi=0$ with $\psi \notin L^{2}\left(\mathbb{R}^{n}\right)$ satisfying other dimension-specific criteria. In dimension $n=1,2$, a resonance occurs if $\psi \in L^{p}\left(\mathbb{R}^{2}\right)$ for some $p>2$, while in dimensions $n=3,4$, the condition 
is instead $(1+|x|)^{-\sigma} \psi \in L^{2}$ for certain values of $\sigma>0$. Working in dimension $n \geq 5$ ensures that $(-\Delta)^{-1} V$ is a bounded operator on $L^{2}\left(\mathbb{R}^{n}\right)$, and forces zero to be an eigenvalue of $H$ if it is not a regular point of the spectrum. In order to handle the low-energy contribution, one typically assumes that zero is a regular point of the spectrum of $H$. Our goal in this paper is to characterize the evolution in odd dimensions $n \geq 5$ when that assumption fails. The analogous case in even dimensions $n \geq 6$ is treated in a separate work [12].

It is known that in general obstructions at zero lead to a loss of time decay in the dispersive estimate. Jensen and Kato [18] showed that in three dimensions, if there is a resonance at zero energy then the propagator $e^{i t H} P_{a c}(H)$ (as an operator between polynomially weighted $L^{2}\left(\mathbb{R}^{3}\right)$ spaces) has leading order decay of $|t|^{-\frac{1}{2}}$ instead of $|t|^{-\frac{3}{2}}$. The same effect occurs if zero is an eigenvalue, despite the fact that $P_{a c}(H)$ explicitly projects away from the associated eigenspace. For all $n \geq 5$, Jensen [16] obtains leading order decay at the rate $|t|^{2-\frac{n}{2}}$ as an operator on weighted $L^{2}\left(\mathbb{R}^{n}\right)$ spaces if zero is an eigenvalue. The subsequent terms of the asymptotic expansion have decay rates $|t|^{1-\frac{n}{2}}$ and $|t|^{-\frac{n}{2}}$ and map between more heavily weighted $L^{2}\left(\mathbb{R}^{n}\right)$ spaces. We are able to recover the same structure of time decay with respect to mappings from $L^{1}\left(\mathbb{R}^{n}\right)$ to $L^{\infty}\left(\mathbb{R}^{n}\right)$, with a finite rank leading order term and a remainder that belongs to weighted spaces. Our results imply Jensen's results by embedding $(1+|x|)^{-\sigma} L^{2}\left(\mathbb{R}^{n}\right) \subset L^{1}\left(\mathbb{R}^{n}\right)$ and $L^{\infty}\left(\mathbb{R}^{n}\right) \subset(1+|x|)^{\sigma} L^{2}\left(\mathbb{R}^{n}\right)$ for $\sigma>\frac{n}{2}$, and in fact our results imply $L^{2}$ estimates with smaller weights than required in [16].

Perhaps the most novel result we prove is that (1) is once again satisfied, with no weights, provided the zero-energy eigenfunctions satisfy the two orthogonality conditions stated in Theorem 1.2 part (3).

The orthogonality conditions mentioned above are directly tied to the spatial decay of eigenfunctions solving $H \psi=0$. For a "generic" eigenfunction, $|\psi(x)| \sim|x|^{2-n}$ for large $x$, a property inherited from the Green's function of the Laplacian. Vanishing moments of $V \psi$ are associated with faster decay of $\psi(x)$, more specifically the condition $\int_{\mathbb{R}^{n}} V \psi d x=0$ in Theorem 1.2 implies that $|\psi(x)| \lesssim|x|^{1-n}$, and if $\int_{\mathbb{R}^{n}} x_{j} V \psi d x=0$ as well for each $j=1 \ldots n$ then in fact $|\psi(x)| \lesssim|x|^{-n}$. At the same time these conditions also cause some leading-order terms of the expansion in [16] to vanish. We show that a more subtle cancellation takes place in the remainder terms, which is why they can be stated with reduced weights as in Theorem 1.2 part (22), or no weights as in part (3) of the same theorem. The first author proved a similar result in three dimensions under the condition $\psi \in L^{1}\left(\mathbb{R}^{3}\right)$, [1] in place of the orthogonality conditions. 
We emphasize that the threshold spectral properties of a given Schrödinger operator $H=-\Delta+V$ are difficult to discern from examing the potential $V$ alone. Eigenvalues at zero can be ruled out if the potential has a lower bound $V(x) \geq-C_{0}\left(1+|x|^{2}\right)^{-1}$, where the constant $C_{0}$ can be determined from the corresponding Hardy inequality. However for potentials with large negative part there is no known simple test to determine whether an eigenvalue is present at zero.

At the end of Section 5 we describe a large family of compactly supported potentials that possess a zero-energy eigenfunction with prescribed polynomial decay at infinity. We believe that the eigenspace is typically one-dimensional, in which case the orthogonality conditions of Theorem 1.2 are satisfied. The construction and supporting arguments are adapted from [11].

To state our main results, first choose a smooth cut-off function $\chi(\lambda)$ with $\chi(\lambda)=1$ if $\lambda<\lambda_{1} / 2$ and $\chi(\lambda)=0$ if $\lambda>\lambda_{1}$, for a sufficiently small $0<\lambda_{1} \ll 1$. In addition, we use the notation $\langle x\rangle:=(1+|x|)$, and define the (polynomially) weighted $L^{p}$ spaces

$$
\|f\|_{L^{p, \sigma}}:=\left\|\langle x\rangle^{\sigma} f\right\|_{p}
$$

and the abbreviations $a-:=a-\epsilon$ and $a+:=a+\epsilon$ for a small, but fixed, $\epsilon>0$.

We prove the following low energy bounds.

Theorem 1.1. Assume that $n \geq 5$ is odd, $|V(x)| \lesssim\langle x\rangle^{-\beta}$ for some $\beta>n$ and that zero is not an eigenvalue of $H=-\Delta+V$ on $\mathbb{R}^{n}$. Then,

$$
\left\|e^{i t H} \chi(H) P_{a c}(H)\right\|_{L^{1} \rightarrow L^{\infty}} \lesssim|t|^{-\frac{n}{2}} .
$$

Theorem 1.2. Assume that $n \geq 5$ is odd, $|V(x)| \lesssim\langle x\rangle^{-\beta}$, and that zero is an eigenvalue of $H=-\Delta+V$ on $\mathbb{R}^{n}$. The low energy Schrödinger propagator $e^{i t H} \chi(H) P_{a c}(H)$ possesses the following structure:

(1) Suppose that there exists $\psi \in \operatorname{Null} H$ such that $\int_{\mathbb{R}^{n}} V \psi d x \neq 0$. Then there is a rank one time dependent operator $\left\|F_{t}\right\|_{L^{1} \rightarrow L^{\infty}} \lesssim|t|^{2-\frac{n}{2}}$ such that for $|t|>1$,

$$
e^{i t H} \chi(H) P_{a c}(H)-F_{t}=\mathcal{E}_{1}(t) .
$$

Where $\left\|\mathcal{E}_{1}\right\|_{L^{1} \rightarrow L^{\infty}}=o\left(|t|^{2-\frac{n}{2}}\right)$ if $\beta>n$ and $\left\|\mathcal{E}_{1}\right\|_{L^{1} \rightarrow L^{\infty}}=O\left(|t|^{1-\frac{n}{2}}\right)$ if $\beta>n+4$.

(2) Suppose that $\int_{\mathbb{R}^{n}} V \psi d x=0$ for each $\psi \in \operatorname{Null} H$ but $\int_{\mathbb{R}^{n}} x_{j} V \psi d x \neq 0$ for some $\psi$ and some $j \in[1, \ldots, n]$. Then there exists a finite rank time dependent operator $G_{t}$ satisfying $\left\|G_{t}\right\|_{L^{1} \rightarrow L^{\infty}} \lesssim|t|^{1-\frac{n}{2}}$ such that for $|t|>1$,

$$
e^{i t H} \chi(H) P_{a c}(H)-G_{t}=\mathcal{E}_{2}(t) .
$$


Where $\left\|\mathcal{E}_{2}\right\|_{L^{1} \rightarrow L^{\infty}}=O\left(|t|^{1-\frac{n}{2}}\right)$ and $\left\|\mathcal{E}_{2}\right\|_{L^{1,0+} \rightarrow L^{\infty, 0-}}=o\left(|t|^{1-\frac{n}{2}}\right)$ if $\beta>n+4$ and $\left\|\mathcal{E}_{2}\right\|_{L^{1,1} \rightarrow L^{\infty,-1}}=O\left(|t|^{-\frac{n}{2}}\right)$ if $\beta>n+8$.

(3) Suppose $\beta>n+8$ and that $\int_{R^{n}} V \psi d x=0$ and $\int_{R^{n}} x_{j} V \psi d x=0$ for all $\psi \in$ Null $H$ and all $j \in[1, \ldots, n]$. Then

$$
\left\|e^{i t H} \chi(H) P_{a c}(H)\right\|_{L^{1} \rightarrow L^{\infty}} \lesssim|t|^{-\frac{n}{2}} .
$$

We note that the assumption that $\int_{\mathbb{R}^{n}} V \psi d x=0$ for each $\psi \in$ Null $H$ is equivalent to assuming that the operator $P_{e} V 1=0$ with $P_{e}$ the projection onto the zero-energy eigenspace. Further, $\int_{R^{n}} x_{j} V \psi d x=0$ for each $j=1,2, \ldots, n$ is equivalent to assuming the operator $P_{e} V x=0$.

These results are fashioned similarly to the asymptotic expansions in [16], with particular emphasis on the behavior of the resolvent of $H$ at low energy. If one assumes greater decay of the potential, then it becomes possible to carry out the resolvent expansion to a greater number of terms, which permits a more detailed description of the time decay of $e^{i t H} \chi(H) P_{a c}(H)$. We note that while $F_{t}$ and $G_{t}$ above have a concise construction, expressions for higher order terms in the expansion are unwieldy enough to discourage writing out an exact formula.

The power series statement of the main theorem is as follows.

Corollary 1.3. If $|V(x)| \lesssim\langle x\rangle^{-n-8-}$, and there is an eigenvalue of $H$ at zero energy, then we have the operator-valued expansion

$$
e^{i t H} \chi(H) P_{a c}(H)=C_{n}|t|^{2-\frac{n}{2}} P_{e} V 1 V P_{e}+|t|^{1-\frac{n}{2}} A_{-1}+|t|^{-\frac{n}{2}} A_{0}(t) .
$$

There exist uniform bounds for $P_{e} V 1 V P_{e}: L^{1} \rightarrow L^{\infty}, A_{-1}: L^{1} \rightarrow L^{\infty}$, and $A_{0}(t)$ : $L^{1,2} \rightarrow L^{\infty,-2}$. The operator $P_{e} V 1 V P_{e}$ is a rank one projection and $A_{-1}$ is finite rank. Furthermore, if $P_{e} V 1=0$, then $A_{0}(t): L^{1,1} \rightarrow L^{\infty,-1}$. If $P_{e} V 1=0$ and $P_{e} V x=0$ then $A_{-1}$ vanishes and $A_{0}(t): L^{1} \rightarrow L^{\infty}$ uniformly in $t$.

We note that this expansion could continue indefinitely in powers of $|t|^{-\frac{n}{2}-k}, k \in \mathbb{N}$. The operators would be finite rank between successively more heavily weighted spaces and would require more decay on the potential $V$, we do not pursue this issue.

Global dispersive estimates are known in all lower dimensions when zero is not a regular point of the spectrum. They are due to the first author and Schlag [13] in one dimension, the second author and Erdoğan [7] in two dimensions, Yajima [29] and Erdoğan-Schlag [9, 8] in three dimensions, and to Erdoğan and the authors [5] in four dimensions. Except for the last 
of these, the low-energy argument builds upon the series expansion for resolvents set forth in [18, 17, 19. We continue to follow this line of argument and work with high-dimensional resolvent expansions similar to those in [16].

We note that the estimates we prove can be combined with the large energy estimates in, for example, [30, 10] to prove analogous statements for the full evolution $e^{i t H} P_{a c}(H)$ without the low-energy cut-off. This requires more assumptions on the the potential, that its polynomially weighted Fourier transform satisfies

$$
\mathcal{F}\left(\langle x\rangle^{2 \sigma} V\right) \in L^{n_{*}}\left(\mathbb{R}^{n}\right) \quad \text { for } \sigma>\frac{1}{n_{*}}=\frac{n-2}{n-1} .
$$

Roughly speaking, this corresponds to having more than $\frac{n-3}{2}+\frac{n-3}{n-2}$ derivatives of $V$ in $L^{2}$.

In addition there has been work on the $L^{p}$ boundedness of the wave operators, which are defined by strong limits on $L^{2}\left(\mathbb{R}^{n}\right)$,

$$
W_{ \pm}=s^{-} \lim _{t \rightarrow \pm \infty} e^{i t H} e^{i t \Delta}
$$

The $L^{p}$ boundedness of the wave operators is of interest to our line of inquiry because of the 'intertwining property'

$$
f(H) P_{a c}=W_{ \pm} f(-\Delta) W_{ \pm}^{*}
$$

which is valid for Borel functions $f$. In dimensions $n \geq 5$, boundedness of the wave operators on $L^{p}$ for $\frac{n}{n-2}<p<\frac{n}{2}$ in the presence of an eigenvalue at zero was established by Yajima [30. in odd dimensions, and Finco-Yajima [10] in even dimensions. In particular, these results imply the mapping estimate

$$
\left\|e^{i t H} P_{a c}(H)\right\|_{L^{p} \rightarrow L^{p^{\prime}}} \lesssim|t|^{-\frac{n}{2}+\frac{n}{p}}
$$

Here $p^{\prime}$ is the conjugate exponent satisfying $\frac{1}{p}+\frac{1}{p^{\prime}}=1$. Roughly speaking, the wave operator results yield a time decay rate of $|t|^{-\frac{n}{2}+2+}$. Similar results in lower dimensions can be found in $[29,20]$.

The fact that $n \geq 5$ allows for greater uniformity of approach, as there are no special dimension-specific considerations related to the distinction between resonances and eigenvalues at zero. There are, however, significant differences in the low-energy expansion of the resolvent depending on whether $n$ is even or odd. While the dispersive bounds stated in Theorem 1.2 hold for all $n \geq 5$, there are not enough shared elements in the computation to treat the even and odd dimensional cases side by side. The present paper considers odd $n$. The case of even $n$ is more technically challenging due to the logarithmic behavior of the resolvent operators and is considered in the companion paper, [12]. 
We define the limiting resolvent operators

$$
R_{V}^{ \pm}\left(\lambda^{2}\right)=\lim _{\epsilon \rightarrow 0^{+}}\left(-\Delta+V-\left(\lambda^{2} \pm i \epsilon\right)\right)^{-1} .
$$

These operators are well-defined on certain weighted $L^{2}\left(\mathbb{R}^{n}\right)$ spaces, see [2]. In fact, there is a zero energy eigenvalue precisely when this operator becomes unbounded as $\lambda \rightarrow 0$.

As usual (cf. [25, 13, 26]), the dispersive estimates follow by considering the operator $e^{i t H} \chi(H) P_{a c}(H)$ as an element of the functional calculus of $H$. Using the Stone formula, we have

$$
e^{i t H} \chi(H) P_{a c}(H) f(x)=\frac{1}{2 \pi i} \int_{0}^{\infty} e^{i t \lambda^{2}} \lambda \chi(\lambda)\left[R_{V}^{+}\left(\lambda^{2}\right)-R_{V}^{-}\left(\lambda^{2}\right)\right] f(x) d \lambda,
$$

with the difference of resolvents $R_{V}^{ \pm}(\lambda)$ providing the absolutely continuous spectral measure. For $\lambda>0$ (and if also at $\lambda=0$ if zero is a regular point of the spectrum) the resolvents are well-defined on certain weighted $L^{2}$ spaces. The key issue when zero energy is not regular is to control the singularities in the spectral measure as $\lambda \rightarrow 0$.

Here $R_{V}^{ \pm}\left(\lambda^{2}\right)$ are operators whose integral kernel we write as $R_{V}^{ \pm}\left(\lambda^{2}\right)(x, y)$. That is, the action of the operator is defined by

$$
R_{V}^{ \pm}\left(\lambda^{2}\right) f(x)=\int_{\mathbb{R}^{n}} R_{V}^{ \pm}\left(\lambda^{2}\right)(x, y) f(y) d y .
$$

The analysis in this paper focuses on bounding the oscillatory integral

$$
\int_{0}^{\infty} e^{i t \lambda^{2}} \lambda \chi(\lambda)\left[R_{V}^{+}\left(\lambda^{2}\right)-R_{V}^{-}\left(\lambda^{2}\right)\right](x, y) d \lambda
$$

in terms of $x, y$ and $t$. A uniform bound of the form $\sup _{x, y} \mid\left(\left.[3)|\lesssim| t\right|^{-\alpha}\right.$ would give us an estimate on $e^{i t H} P_{a c}(H)$ as an operator from $L^{1} \rightarrow L^{\infty}$. We leave open the option of dependence on $x$ and $y$ to allow for estimates between weighted $L^{1}$ and weighted $L^{\infty}$ spaces. That is, an estimate of the form $|(\sqrt[3]{3})| \lesssim|t|^{-\alpha}\langle x\rangle^{\sigma}\langle y\rangle^{\sigma^{\prime}}$ yields an estimate as an operator from $L^{1, \sigma^{\prime}} \rightarrow L^{\infty,-\sigma}$.

Accordingly, we study expansions for the resolvent operators $R_{V}^{ \pm}\left(\lambda^{2}\right)$ in a neighborhood of zero. The type of terms present is heavily influenced by whether $n$ is even or odd. In odd dimensions the expansion is a formal Laurent series

$$
R_{V}^{ \pm}\left(\lambda^{2}\right)=A \lambda^{-2}+B \lambda^{-1}+O(1)
$$

with operator-valued coefficients. The operators $A$ and $B$ are zero if there are no zero-energy eigenvalues (or resonances in dimensions $n=1,3$ ). In even dimensions the expansions are more complicated, involving terms of the form $\lambda^{k}(\log \lambda)^{\ell}, k \geq-2$, see for example [16, 17, 7, 5, 12. 
The organization of the paper is as follows. We begin in Section 2 by developing expansions for the free resolvent and develop necessary machinery to understand the spectral measure $E^{\prime}(\lambda)=\frac{1}{2 \pi i}\left[R_{V}^{+}\left(\lambda^{2}\right)-R_{V}^{-}\left(\lambda^{2}\right)\right]$. In Section 3 we prove dispersive estimates for the tail of the Born series, (27), which is the portion of the evolution that is sensitive to the existence of zero-energy eigenvalues. Next, in Section 4, we prove dispersive estimates for the finite Born series series, (26), which is the portion of the low energy evolution that is unaffected by zero-energy eigenvalues. Collectively these form a proof of Theorem 1.2. In Section 5 we provide a characterization of the spectral subspaces of $L^{2}$ related to the zero energy eigenspace. Finally, Section [6 contains an index of technical integral estimates that arise in the course of the preceding calculations.

\section{Resolvent Expansions Around Zero}

In this section we first develop expansions for the integral kernels of the free resolvents $R_{0}^{ \pm}\left(\lambda^{2}\right):=\lim _{\epsilon \rightarrow 0^{+}}\left(-\Delta-\left(\lambda^{2} \pm i \epsilon\right)\right)^{-1}$ to understand the pertubed resolvent operators $R_{V}^{ \pm}\left(\lambda^{2}\right):=\lim _{\epsilon \rightarrow 0^{+}}\left(-\Delta+V-\left(\lambda^{2} \pm i \epsilon\right)\right)^{-1}$ with the aim of understanding the spectral measure in (3).

In developing these expansions we employ the following notation

$$
f(\lambda)=\widetilde{O}(g(\lambda))
$$

to indicate that

$$
\frac{d^{j}}{d \lambda^{j}} f(\lambda)=O\left(\frac{d^{j}}{d \lambda^{j}} g(\lambda)\right)
$$

If the relationship holds only for the first $k$ derivatives, we use the notation $f(\lambda)=\widetilde{O}_{k}(g(\lambda))$. With a slight abuse of notation, we may write $f(\lambda)=\widetilde{O}\left(\lambda^{k}\right)$ for an integer $k$, to indicate that $\frac{d^{j}}{d \lambda^{j}} f(\lambda)=O\left(\lambda^{k-j}\right)$. This distinction is particularly important for when $k \geq 0$ and $j>k$.

Writing the free resolvent kernel in terms of the Hankel functions we have

$$
R_{0}(z)(x, y)=\frac{i}{4}\left(\frac{z^{1 / 2}}{2 \pi|x-y|}\right)^{\frac{n}{2}-1} H_{\frac{n}{2}-1}^{(1)}\left(z^{1 / 2}|x-y|\right) .
$$

Here $H_{\frac{n}{2}-1}^{(1)}(\cdot)$ is the Hankel function of the first kind. Since $n$ is odd, these are Hankel functions of half-integer order, which can be expressed in closed form. We use the following explicit representation for the kernel of the limiting resolvent operators $R_{0}^{ \pm}\left(\lambda^{2}\right)$ (see, e.g., [16])

$$
R_{0}^{ \pm}\left(\lambda^{2}\right)(x, y)=\mathcal{G}_{n}( \pm \lambda,|x-y|)
$$


where

$$
\mathcal{G}_{n}(\lambda, r)=C_{n} \frac{e^{i \lambda r}}{r^{n-2}} \sum_{\ell=0}^{\frac{n-3}{2}} \frac{(n-3-\ell) !}{\ell !\left(\frac{n-3}{2}-\ell\right) !}(-2 i r \lambda)^{\ell}
$$

For small $\lambda$, one can expand these in a Taylor series, as in Lemma 3.5 of [16] to see with $G_{j}(x, y)=c_{j}|x-y|^{2+j-n}$ with $c_{j}$ real-valued constants.

$$
\mathcal{G}_{n}(\lambda, r)=G_{0}+\sum_{j=1}^{\frac{n-3}{2}} \lambda^{2 j} G_{2 j}+i \lambda^{n-2} G_{n-2}+\lambda^{n-1} G_{n-1}+i \lambda^{n} G_{n}+E(\lambda), \text { as } \lambda \rightarrow 0 .
$$

Where the error term $E(\lambda)=O\left(\lambda^{n+1}\right)$ is understood as a Hilbert-Schmidt operator between weighted $L^{2}$ spaces. We can (and need to) be more delicate with this error term.

It is quite natural to view this as an operator between weighted $L^{2}$ spaces as $G_{0}$ is a scalar multiple of the fractional integral operator $I_{2}$ whereas the remaining terms are either scalar multiples of the fractional integral operators $I_{2 j+2}$ or can be bounded in a Hilbert-Schmidt norm with sufficiently large polynomial weights. In particular, we note that

$$
\begin{aligned}
G_{0}(x, y) & =c_{0}|x-y|^{2-n}=(-\Delta)^{-1}(x, y), \\
G_{n-2}(x, y) & =c_{n-2}, \\
G_{n}(x, y) & =c_{n}|x-y|^{2}=c_{n}(x-y) \cdot(x-y)=c_{n}\left[|x|^{2}-2 x \cdot y+|y|^{2}\right],
\end{aligned}
$$

We may also use the notation $G_{n-2}=c_{n-2} 1$, where 1 indicates the operator with kernel $1(x, y)=1$.

Lemma 2.1. For $\lambda \leq \lambda_{1}$, we have the expansion(s) for the free resolvent,

$$
\begin{aligned}
R_{0}^{ \pm}\left(\lambda^{2}\right) & =G_{0}+\sum_{j=1}^{\frac{n-3}{2}} \lambda^{2 j} G_{2 j} \pm i \lambda^{n-2} G_{n-2}+E_{0}^{ \pm}(\lambda) \\
& =G_{0}+\sum_{j=1}^{\frac{n-3}{2}} \lambda^{2 j} G_{2 j} \pm i \lambda^{n-2} G_{n-2}+\lambda^{n-1} G_{n-1}+E_{1}^{ \pm}(\lambda) \\
& =G_{0}+\sum_{j=1}^{\frac{n-3}{2}} \lambda^{2 j} G_{2 j} \pm i \lambda^{n-2} G_{n-2}+\lambda^{n-1} G_{n-1} \pm i \lambda^{n} G_{n}+E_{2}^{ \pm}(\lambda) \\
& =G_{0}+\sum_{j=1}^{\frac{n-3}{2}} \lambda^{2 j} G_{2 j} \pm i \lambda^{n-2} G_{n-2}+\lambda^{n-1} G_{n-1} \pm i \lambda^{n} G_{n}+\lambda^{n+1} G_{n+1} \\
& \pm i \lambda^{n+2} G_{n+2}+E_{3}^{ \pm}(\lambda) .
\end{aligned}
$$


Where, for any $0 \leq \ell \leq 1$,

$$
\begin{aligned}
& E_{0}^{ \pm}(\lambda)=|x-y|^{\ell} \widetilde{O}_{\frac{n-1}{2}}\left(\lambda^{n-2+\ell}\right), \\
& E_{1}^{ \pm}(\lambda)=|x-y|^{1+\ell} \widetilde{O}_{\frac{n+1}{2}}\left(\lambda^{n-1+\ell}\right), \\
& E_{2}^{ \pm}(\lambda)=|x-y|^{2+\ell} \widetilde{O}_{\frac{n+3}{2}}\left(\lambda^{n+\ell}\right), \\
& E_{3}^{ \pm}(\lambda)=|x-y|^{4+\ell} \widetilde{O}_{\frac{n+7}{2}}\left(\lambda^{n+2+\ell}\right) .
\end{aligned}
$$

Proof. Using a Taylor series expansion on (5) when $\lambda|x-y| \leq \frac{1}{2}$, one has

$$
\begin{aligned}
\mathcal{G}_{n}(\lambda, r) & =G_{0}+\sum_{j=1}^{\frac{n-3}{2}} \lambda^{2 j} G_{2 j}+i \lambda^{n-2} G_{n-2}+\lambda^{n-1} G_{n-1}+i \lambda^{n} G_{n}+\lambda^{n+1} G_{n+1} \\
& +i \lambda^{n+2} G_{n+2}+\widetilde{O}\left(\lambda^{n-2}(\lambda|x-y|)^{5}\right) .
\end{aligned}
$$

This expansion can be truncated earlier, using $G_{j}(x, y)=c_{j}|x-y|^{n-2+j}$ and $\lambda|x-y| \lesssim 1$.

On the other hand, if $\lambda|x-y| \gtrsim 1$ we note that differentiation in $\lambda$ in (5) is comparable to either division by $\lambda$ or multiplication by $|x-y|$. So that, for $0 \leq k \leq \frac{n-1}{2}$,

$$
\left|\partial_{\lambda}^{k} R_{0}^{ \pm}\left(\lambda^{2}\right)(x, y)\right| \lesssim\left(\frac{1}{|x-y|^{n-2}}+\frac{\lambda^{\frac{n-3}{2}}}{|x-y|^{\frac{n-1}{2}}}\right)\left(\lambda^{-k}+|x-y|^{k}\right) \lesssim \lambda^{n-2-k} .
$$

Here we use that $|x-y|^{-1} \lesssim \lambda$. Using that $\lambda|x-y| \gtrsim 1$, can can gain more $\lambda$ smallness at the cost of growth in $|x-y|$. Specifically, for any $\ell \geq 0$ and $0 \leq k \leq \frac{n-1}{2}$,

$$
\left|\partial_{\lambda}^{k} R_{0}^{ \pm}\left(\lambda^{2}\right)(x, y)\right| \lesssim \lambda^{n-2-k}(\lambda|x-y|)^{\ell} .
$$

For the first expansion we take

$$
E_{0}^{ \pm}(\lambda)=R_{0}^{ \pm}\left(\lambda^{2}\right)-G_{0}-\sum_{j=1}^{\frac{n-3}{2}} \lambda^{2 j} G_{2 j} \mp i \lambda^{n-2} G_{n-2} .
$$

The error bounds if $\lambda|x-y| \leq \frac{1}{2}$ are clear from expanding (5) in a Taylor series. On the other hand, if $\lambda|x-y| \gtrsim 1$ we have that

$$
\left|\partial_{\lambda}^{k}\left(G_{0}+\sum_{j=1}^{\frac{n-3}{2}} \lambda^{2 j} G_{2 j} \mp i \lambda^{n-2} G_{n-2}\right)\right| \lesssim \lambda^{n-2-k} .
$$

Where one uses that $|x-y|^{-1} \lesssim \lambda$ in this case. The remaining error terms $E_{1}^{ \pm}(\lambda)$ and $E_{2}^{ \pm}(\lambda)$ arise from subtracting more Taylor terms from the free resolvent.

$$
E_{1}^{ \pm}(\lambda)=R_{0}^{ \pm}\left(\lambda^{2}\right)-G_{0}-\sum_{j=1}^{\frac{n-3}{2}} \lambda^{2 j} G_{2 j} \mp i \lambda^{n-2} G_{n-2}-\lambda^{n-1} G_{n-1},
$$




$$
E_{2}^{ \pm}(\lambda)=R_{0}^{ \pm}\left(\lambda^{2}\right)-G_{0}-\sum_{j=1}^{\frac{n-3}{2}} \lambda^{2 j} G_{2 j} \mp i \lambda^{n-2} G_{n-2}-\lambda^{n-1} G_{n-1} \mp i \lambda^{n} G_{n},
$$

and

$$
\begin{aligned}
E_{3}^{ \pm}(\lambda)=R_{0}^{ \pm}\left(\lambda^{2}\right)-G_{0}-\sum_{j=1}^{\frac{n-3}{2}} \lambda^{2 j} G_{2 j} \mp i \lambda^{n-2} G_{n-2} & -\lambda^{n-1} G_{n-1} \mp i \lambda^{n} G_{n} \\
& -\lambda^{n+1} G_{n+1} \mp i \lambda^{n+2} G_{n+2} .
\end{aligned}
$$

In particular, we note that for $k \leq n-2+j$,

$$
\left|\partial_{\lambda}^{k} \lambda^{n-2+j} G_{n-2+j}\right| \lesssim \lambda^{n-2+j-k} G_{n-2+j} \lesssim \lambda^{n-2+j-k}|x-y|^{j} .
$$

With the bound being zero if $k>n-2+j$. As before, the bounds are clear for $\lambda|x-y| \leq \frac{1}{2}$, for $\lambda|x-y| \gtrsim 1$, one has for any $\ell \geq 0$

$$
\left|\partial_{\lambda}^{k} \lambda^{n-2+j} G_{n-2+j}\right| \lesssim \lambda^{n-2+j-k}|x-y|^{j}(\lambda|x-y|)^{\ell}
$$

Finally, we note that if $\lambda|x-y| \gtrsim 1$ and $k \geq \frac{n+3}{2}$ when differentiating $R_{0}^{ \pm}\left(\lambda^{2}\right)$, multiplication by $|x-y|$ dominates division by $\lambda$, thus we have

$$
\left|\partial_{\lambda}^{k} R_{0}^{ \pm}\left(\lambda^{2}\right)(x, y)\right| \lesssim \frac{\lambda^{\frac{n-3}{2}}}{|x-y|^{\frac{n-1}{2}}}\left(\lambda^{-k}+|x-y|^{k}\right) \lesssim \lambda^{\frac{n-3}{2}}|x-y|^{k-\frac{n-1}{2}}(\lambda|x-y|)^{\ell} .
$$

This suffices to prove the bounds for $E_{j}^{ \pm}(\lambda)$.

It is important to note that the kernels of the operators $G_{j}$ are strictly real-valued. The hypotheses of the lemma below are not optimal, but suffice for our purposes. We give the proof of this Lemma in Section 6.

Lemma 2.2. If $|V(x)| \lesssim\langle x\rangle^{-\frac{n+1}{2}-}, \sigma>\frac{1}{2}$ and $\kappa \geq \frac{n-3}{4}$, then

$$
\left\|\left(R_{0}^{ \pm}(\lambda)^{2} V\right)^{\kappa-1}(y, \cdot) R_{0}(\cdot, x)\right\|_{L_{y}^{2,-\sigma}} \lesssim\langle\lambda\rangle^{\kappa\left(\frac{n-3}{2}\right)} .
$$

uniformly in $x$.

Define the operator $U: L^{2} \rightarrow L^{2}$ by its kernel

$$
U(x)= \begin{cases}1 & V(x) \geq 0 \\ -1 & V(x)<0\end{cases}
$$

That is, $U$ is the sign of $V$. Further define $v=|V|^{\frac{1}{2}}, w=U v$ and

$$
M^{ \pm}(\lambda)=U+v R_{0}^{ \pm}\left(\lambda^{2}\right) v
$$


We wish to use the symmetric resolvent identity,

$$
R_{V}^{ \pm}\left(\lambda^{2}\right)=R_{0}^{ \pm}\left(\lambda^{2}\right)-R_{0}^{ \pm}\left(\lambda^{2}\right) v M^{ \pm}(\lambda)^{-1} v R_{0}^{ \pm}\left(\lambda^{2}\right),
$$

which is valid for $\Im(\lambda)>0$, to understand the spectral measure in (3).

Lemma 2.2 allows us to make sense of the symmetric resolvent identity, provided $|V(x)| \lesssim$ $\langle x\rangle^{-\frac{n+1}{2}-}$, by iterating the standard resolvent identity

$$
R_{V}^{ \pm}\left(\lambda^{2}\right)=R_{0}^{ \pm}\left(\lambda^{2}\right)-R_{0}^{ \pm}\left(\lambda^{2}\right) V R_{V}^{ \pm}\left(\lambda^{2}\right)=R_{0}^{ \pm}\left(\lambda^{2}\right)-R_{V}^{ \pm}\left(\lambda^{2}\right) V R_{0}^{ \pm}\left(\lambda^{2}\right)
$$

on both sides of $M^{ \pm}(\lambda)^{-1}$ in (10) a sufficient number of times to get to a local $L^{2}$ space, from which multiplication by $v$ takes the iterated resolvents to $L^{2}$.

Our main tool used to invert $M^{ \pm}(\lambda)=U+v R_{0}^{ \pm}\left(\lambda^{2}\right) v$ for small $\lambda$, is the following lemma (see Lemma 2.1 in [19]).

Lemma 2.3. Let $A$ be a closed operator on a Hilbert space $\mathcal{H}$ and $S$ a projection. Suppose $A+S$ has a bounded inverse. Then $A$ has a bounded inverse if and only if

$$
B:=S-S(A+S)^{-1} S
$$

has a bounded inverse in $S \mathcal{H}$, and in this case

$$
A^{-1}=(A+S)^{-1}+(A+S)^{-1} S B^{-1} S(A+S)^{-1} .
$$

Following the terminology used in [26, 7, [5],

Definition 2.4. We say an operator $K: L^{2}\left(\mathbb{R}^{n}\right) \rightarrow L^{2}\left(\mathbb{R}^{n}\right)$ with kernel $K(\cdot, \cdot)$ is absolutely bounded if the operator with kernel $|K(\cdot, \cdot)|$ is bounded from $L^{2}\left(\mathbb{R}^{n}\right)$ to $L^{2}\left(\mathbb{R}^{n}\right)$.

We recall the definition of the Hilbert-Schmidt norm of an operator $K$ with integral kernel $K(x, y)$,

$$
\|K\|_{H S}=\left(\iint_{\mathbb{R}^{2 n}}|K(x, y)|^{2} d x d y\right)^{\frac{1}{2}} .
$$

We note that Hilbert-Schmidt and finite rank operators are immediately absolutely bounded.

Lemma 2.5. Assuming that $v(x) \lesssim\langle x\rangle^{-\beta}$. If $\beta>\frac{n}{2}+\ell$, then for $0 \leq \ell \leq 1$, we have

$$
M^{ \pm}(\lambda)=U+v G_{0} v+\sum_{j=1}^{\frac{n-3}{2}} \lambda^{2 j} v G_{2 j} v \pm i \lambda^{n-2} v G_{n-2} v+M_{0}^{ \pm}(\lambda),
$$


Where the kernels of the operators $G_{j}$ are absolutely bounded with real-valued kernels. Further,

$$
\sum_{j=0}^{\frac{n-1}{2}}\left\|\sup _{0<\lambda<\lambda_{1}} \lambda^{j+2-n-\ell} \partial_{\lambda}^{j} M_{0}^{ \pm}(\lambda)\right\|_{H S} \lesssim 1 .
$$

If $\beta>\frac{n}{2}+2+\ell$, then

$$
M_{0}^{ \pm}(\lambda)=\lambda^{n-1} v G_{n-1} v \pm i \lambda^{n} v G_{n} v+M_{1}^{ \pm}(\lambda)
$$

with

$$
\sum_{j=0}^{\frac{n+1}{2}}\left\|\sup _{0<\lambda<\lambda_{1}} \lambda^{j-n-\ell} \partial_{\lambda}^{j} M_{1}^{ \pm}(\lambda)\right\|_{H S} \lesssim 1 .
$$

If $\beta>\frac{n}{2}+4+\ell$, then

$$
M_{1}^{ \pm}(\lambda)=\lambda^{n+1} v G_{n+1} v \pm i \lambda^{n+2} v G_{n+2} v+M_{2}^{ \pm}(\lambda)
$$

with

$$
\sum_{j=0}^{\frac{n+1}{2}}\left\|\sup _{0<\lambda<\lambda_{1}} \lambda^{j-n-2-\ell} \partial_{\lambda}^{j} M_{2}^{ \pm}(\lambda)\right\|_{H S} \lesssim 1 .
$$

Proof. The proof follows from the definition of the operators $M^{ \pm}(\lambda)$ and the expansion for the free resolvent in Lemma 2.1. The bound on the error terms follows from the fact that if $k>-\frac{n}{2}$ then $\langle x\rangle^{-\beta}|x-y|^{k}\langle y\rangle^{-\beta}$ is Hilbert-Schmidt, and hence absolutely bounded, provided $\beta>\frac{n}{2}+k$. So that the operators $v G_{j} v$ is Hilbert Schmidt for $j \geq \frac{n}{2}-2$ provided $\beta>j+2-\frac{n}{2}$.

Remark 2.6. The error estimates here can be more compactly summarized as

$$
M_{0}^{ \pm}(\lambda)=\widetilde{O}_{\frac{n-1}{2}}\left(\lambda^{n-2+\ell}\right), \quad M_{1}^{ \pm}(\lambda)=\widetilde{O}_{\frac{n+1}{2}}\left(\lambda^{n+\ell}\right), \quad M_{2}^{ \pm}(\lambda)=\widetilde{O}_{\frac{n+1}{2}}\left(\lambda^{n+2+\ell}\right),
$$

as absolutely bounded operators on $L^{2}\left(\mathbb{R}^{n}\right)$, for $0<\lambda<\lambda_{1}$.

We note that $U+v G_{0} v$ is not invertible if there is an eigenvalue at zero, see Lemma 5.1 below. Define $S_{1}$ to be the Riesz projection onto the kernel of $U+v G_{0} v$ as an operator on $L^{2}\left(\mathbb{R}^{n}\right)$. Then the operator $U+v G_{0} v+S_{1}$ is invertible on $L^{2}$, accordingly we define

$$
D_{0}:=\left(U+v G_{0} v+S_{1}\right)^{-1} .
$$

This operator can be seen to be absolutely bounded. 
Lemma 2.7. If $|V(x)| \lesssim\langle x\rangle^{-\frac{n+1}{2}-}$, then the operator $D_{0}$ is absolutely bounded in $L^{2}\left(\mathbb{R}^{n}\right)$. Proof. We have the resolvent identity

$$
D_{0}=U-D_{0}\left(v G_{0} v+S_{1}\right) U
$$

Iterating this identity, we have for $k>1$,

$$
D_{0}=U+\sum_{j=1}^{k-1}(-1)^{j} U\left(\left(v G_{0} v+S_{1}\right) U\right)^{j}+(-1)^{k} D_{0}\left[\left(v G_{0} v+S_{1}\right) U\right]^{k} .
$$

Using Lemma 6.1 as in the proof Lemma 2.2 (see Section [6) one can see that each iteration of $v G_{0} v$ reduces the local singularity by two powers. Thus, we need to iterate the the identities at least $k=\left\lceil\frac{n-3}{4}\right\rceil$ terms to get the final iterated operator to be locally $L^{2}$.

We note that $U$ is clearly absolutely bounded on $L^{2}\left(\mathbb{R}^{n}\right)$. One can note that

$$
0=S_{1}\left(U+v G_{0} v\right) \quad \Rightarrow \quad S_{1} U=-S_{1} v G_{0} v \quad \Rightarrow \quad S_{1}=-S_{1} v G_{0} w .
$$

To see that the mapping properties of $S_{1}$ are at least as good as those of $v G_{0} v$. Alternatively, since $U+v G_{0} v$ is a compact perturbation of the invertible operator $U$, the Fredholm alternative guarantees that the projection operator $S_{1}$ is finite rank.

The operator $G_{0}$ is a scalar multiple of the fractional integral operator $I_{2}$ which is a compact operator on $L^{2, \sigma} \rightarrow L^{2,-\sigma}$ for $\sigma>1$ by Lemma 2.3 of [16]. Then $v G_{0} w$ is absolutely bounded on $L^{2}$ provided $v(x) \lesssim\langle x\rangle^{-1-}$.

So that $D_{0}$ is the sum of absolutely bounded operators, $U$, and a Hilbert-Schmidt operators. Since $D_{0}\left[\left(v G_{0} v\right) U\right]^{k}$ is a bounded operator composed with a Hilbert-Schmidt operator, it is Hilbert-Schmidt.

The above proof is valid whether zero is regular or not. When zero is regular, $S_{1}=0$ so many of the terms considered vanish.

We will apply Lemma 2.3 with $A=M^{ \pm}(\lambda)$ and $S=S_{1}$, the Riesz projection onto the kernel of $U+v G_{0} v$. Thus, we need to show that $M^{ \pm}(\lambda)+S_{1}$ has a bounded inverse in $L^{2}\left(\mathbb{R}^{n}\right)$ and

$$
B_{ \pm}(\lambda)=S_{1}-S_{1}\left(M^{ \pm}(\lambda)+S_{1}\right)^{-1} S_{1}
$$

has a bounded inverse in $S_{1} L^{2}\left(\mathbb{R}^{n}\right)$.

Lemma 2.8. Suppose that zero is not a regular point of the spectrum of $H=-\Delta+V$, and let $S_{1}$ be the corresponding Riesz projection on the the zero energy eigenspace. The for 
sufficiently small $\lambda_{1}>0$, the operators $M^{ \pm}(\lambda)+S_{1}$ are invertible for all $0<\lambda<\lambda_{1}$ as bounded operators on $L^{2}\left(\mathbb{R}^{n}\right)$. Further, for any $0 \leq \ell \leq 1$, if $\beta>\frac{n}{2}+\ell$ then we have the following expansions

$$
\left(M^{ \pm}(\lambda)+S_{1}\right)^{-1}=D_{0}+\sum_{j=1}^{\frac{n-3}{2}} \lambda^{2 j} C_{2 j} \mp i \lambda^{n-2} D_{0} v G_{n-2} v D_{0}+\widetilde{M}_{0}^{ \pm}(\lambda)
$$

where $\widetilde{M}_{0}^{ \pm}(\lambda)$ satisfies the same bounds as $M_{0}^{ \pm}(\lambda)$ and the operators $C_{k}$ are absolutely bounded on $L^{2}$ with real-valued kernels. Further, if $\beta>\frac{n}{2}+2+\ell$ then

$$
\widetilde{M}_{0}^{ \pm}(\lambda)=\lambda^{n-1} C_{n-1} \pm i \lambda^{n} C_{n}+\widetilde{M}_{1}^{ \pm}(\lambda)
$$

where $C_{n}=D_{0} v G_{n-2} v D_{0} v G_{2} v D_{0}+D_{0} v G_{2} v D_{0} v G_{n-2} v D_{0}-D_{0} v G_{n} v D_{0}$, and $\widetilde{M}_{1}^{ \pm}(\lambda)$ satisfies the same bounds as $M_{1}^{ \pm}(\lambda)$. Further, if $\beta>\frac{n}{2}+4+\ell$ then

$$
\widetilde{M}_{1}^{ \pm}(\lambda)=\lambda^{n+1} C_{n+1} \pm i \lambda^{n+2} C_{n+2}+\widetilde{M}_{2}^{ \pm}(\lambda)
$$

where $\widetilde{M}_{2}^{ \pm}(\lambda)$ satisfies the same bounds as $M_{2}^{ \pm}(\lambda)$.

Proof. We use a Neumann series expansion. We show the case of $M^{+}$and omit the superscript, the '-' case follows similarly. Using (11) we have

$$
\begin{gathered}
\left(M(\lambda)+S_{1}\right)^{-1}=\left(U+v G_{0} v+S_{1}+\sum_{j=1}^{\frac{n-3}{2}} \lambda^{2 j} v G_{2 j} v+i \lambda^{n-2} v G_{n-2} v+M_{0}(\lambda)\right)^{-1} \\
=D_{0}\left(\mathbb{1}+\sum_{j=1}^{\frac{n-3}{2}} \lambda^{2 j} v G_{2 j} v D_{0}+i \lambda^{n-2} v G_{n-2} v D_{0}+M_{0}(\lambda) D\right)^{-1} \\
=D_{0}-\lambda^{2} D_{0} v G_{2} v D_{0}+\lambda^{4}\left[D_{0} v G_{2} v D_{0} v G_{2} v D_{0}-D_{0} v G_{4} v D_{0}\right]+\sum_{j=3}^{\frac{n-3}{2}} \lambda^{2 j} C_{2 j} \\
-D_{0}\left[i \lambda^{n-2} v G_{n-2} v+M_{0}(\lambda)\right] D_{0}+\lambda^{2}\left[D_{0} v G_{2} v D_{0}\left[i \lambda^{n-2} v G_{n-2} v+M_{0}(\lambda)\right] D_{0}\right. \\
\left.+\lambda^{2} D_{0}\left[i \lambda^{n-2} v G_{n-2} v+M_{0}(\lambda)\right] D_{0} v G_{2} v D_{0}\right]+\lambda^{4}\left[D_{0} v G_{4} v D_{0}\left[i \lambda^{n-2} v G_{n-2} v+M_{0}(\lambda)\right]\right] D_{0} \\
\left.+\lambda^{4} D_{0}\left[i \lambda^{n-2} v G_{n-2} v+M_{0}(\lambda)\right] G_{4} v D_{0}\right]+\widetilde{M}_{2}(\lambda) .
\end{gathered}
$$

One can find explicitly the operators $C_{2 j}$ in terms of $D_{0}$ and the operators $G_{2 k}$, but this is not worth the effort. One only needs that these operators are absolutely bounded with real-valued kernels. These properties are inherited from $D_{0}$ and $v G_{2 k} v$ using that the composition of absolutely bounded operator are absolutely bounded. 
What is important, in our analysis in Section 3 , is the odd powers of $\lambda$. We note that the first odd power of $\lambda$ arises in

$$
\begin{gathered}
-D_{0}\left[i \lambda^{n-2} v G_{n-2} v+M_{0}(\lambda)\right] D_{0}=-D_{0}\left[i \lambda^{n-2} v G_{n-2} v+\lambda^{n-1} v G_{n-1} v+i \lambda^{n} v G_{n} v+M_{1}(\lambda)\right] D_{0} \\
=-i \lambda^{n-2} D_{0} v G_{n-2} v D_{0}-\lambda^{n-1} D_{0} v G_{n-1} v D_{0}-i \lambda^{n} D_{0} v G_{n} v D_{0}-D_{0} M_{1}(\lambda) D_{0}
\end{gathered}
$$

The next odd power occurs from the $i \lambda^{n} D_{0} v G_{n} v D_{0}$ term and the ' $x^{2}$ ' term in the Neumann series, that is the term with both $G_{2}$ and $i \lambda^{n-2} v G_{n-2} v$, accordingly we see that the $\lambda^{n}$ term is given by

$$
i \lambda^{n}\left[D_{0} v G_{n-2} v D_{0} v G_{2} v D_{0}+D_{0} v G_{2} v D_{0} v G_{n-2} v D_{0}-D_{0} v G_{n} v D_{0}\right]
$$

The operators that accompany $\lambda^{n+2}$ are not calculated explicitly, but again are absolutely bounded. The error bounds follow from the bounds in Lemma 2.5 and the Neumann series expansion above.

Remark 2.9. We note here that is zero is regular the above Lemma suffices to establish the dispersive estimates using the techniques in Sections 3 and 4 . In this case, $S_{1}=0$, $D_{0}=\left(U+v G_{0} v\right)^{-1}$ is still absolutely bounded and we have the expansion

$$
M^{ \pm}(\lambda)^{-1}=D_{0}+\sum_{j=1}^{\frac{n-3}{2}} \lambda^{2 j} C_{2 j} \mp i \lambda^{n-2} D_{0} v G_{n-2} v D_{0}+\widetilde{M}_{0}^{ \pm}(\lambda),
$$

with $C_{2 j}$ real-valued, absolutely bounded operators.

Now we turn to the operators $B_{ \pm}(\lambda)$ for use in Lemma 2.3. Recall that

$$
B_{ \pm}(\lambda)=S_{1}-S_{1}\left(M^{ \pm}(\lambda)+S_{1}\right)^{-1} S_{1}
$$

and that $S_{1} D_{0}=D_{0} S_{1}=S_{1}$. Thus

$$
\begin{aligned}
B_{ \pm}(\lambda) & =S_{1}-S_{1}\left[D_{0}+\sum_{j=1}^{\frac{n-3}{2}} \lambda^{2 j} C_{2 j} \mp i \lambda^{n-2} D_{0} v G_{n-2} v D_{0}+\widetilde{M}_{0}^{ \pm}(\lambda)\right] S_{1} \\
& =-\sum_{j=1}^{\frac{n-3}{2}} \lambda^{2 j} S_{1} C_{2 j} S_{1} \pm i \lambda^{n-2} S_{1} v G_{n-2} v S_{1}-S_{1} \widetilde{M}_{0}^{ \pm}(\lambda) S_{1} \\
& =-\lambda^{2} S_{1} v G_{2} v S_{1}-\sum_{j=2}^{\frac{n-3}{2}} \lambda^{2 j} S_{1} C_{2 j} S_{1} \pm i \lambda^{n-2} S_{1} v G_{n-2} v S_{1}-S_{1} \widetilde{M}_{0}^{ \pm}(\lambda) S_{1}
\end{aligned}
$$


So that the invertibility of $B_{ \pm}(\lambda)$ hinges upon the invertibility of the operator $S_{1} D_{0} v G_{2} v D_{0} S_{1}=S_{1} v G_{2} v S_{1}$ on $S_{1} L^{2}\left(\mathbb{R}^{n}\right)$, which is established in Lemma 5.3 below. Thus we define $D_{1}:=\left(S_{1} v G_{2} v S_{1}\right)^{-1}$ as an operator on $S_{1} L^{2}\left(\mathbb{R}^{n}\right)$. Noting that $D_{1}=S_{1} D_{1} S_{1}$, it is clear that $D_{1}$ is absolutely bounded.

Lemma 2.10. We have the following expansions, if $\beta>\frac{n}{2}+\ell$ for any $0 \leq \ell \leq 1$, then

$$
B_{ \pm}(\lambda)^{-1}=-\frac{D_{1}}{\lambda^{2}}+\sum_{j=2}^{\frac{n-3}{2}} \lambda^{2 j-4} B_{2 j} \pm i \lambda^{n-6} D_{1} v G_{n-2} v D_{1}+\widetilde{B}_{0}^{ \pm}(\lambda)
$$

where $\widetilde{B}_{0}^{ \pm}(\lambda)$ satisfies the same bounds as $\lambda^{-4} M_{0}^{ \pm}(\lambda)$ and the operators $B_{k}$ are absolutely bounded on $L^{2}$ with real-valued kernels. Further, if $\beta>\frac{n}{2}+2+\ell$ then

$$
\widetilde{B}_{0}^{ \pm}(\lambda)=\lambda^{n-5} B_{n-1} \pm i \lambda^{n-4} B_{n}+\widetilde{B}_{1}^{ \pm}(\lambda),
$$

where $B_{n}=D_{1} v G_{n-2} v D_{0} v G_{2} v D_{1}+D_{1} v G_{2} v D_{0} v G_{n-2} v D_{1}-D_{1} v G_{n} v D_{1}$ $D_{1} v G_{n-2} v D_{1} C_{4} D_{1}-D_{1} C_{4} D_{1} v G_{n-2} v D_{1}$, and $\widetilde{B}_{1}^{ \pm}(\lambda)$ satisfies the same bounds as $\lambda^{-4} M_{1}^{ \pm}(\lambda)$. Further, if $\beta>\frac{n}{2}+4+\ell$ then

$$
\widetilde{B}_{1}^{ \pm}(\lambda)=\lambda^{n-3} B_{n+1} \pm i \lambda^{n-2} B_{n+2}+\widetilde{B}_{2}^{ \pm}(\lambda),
$$

where $\widetilde{B}_{2}^{ \pm}(\lambda)$ satisfies the same bounds as $\lambda^{-4} M_{2}^{ \pm}(\lambda)$.

Proof. As usual we consider the '+' case and omit subscripts, the '-' case follows similarly. We begin by noting that

$$
\begin{aligned}
& B^{-1}(\lambda)=\left[-\lambda^{2} S_{1} v G_{2} v S_{1}-\sum_{j=2}^{\frac{n-3}{2}} \lambda^{2 j} S_{1} C_{2 j} S_{1} \pm i \lambda^{n-2} S_{1} v G_{n-2} v S_{1}-S_{1} \widetilde{M}_{0}^{ \pm}(\lambda) S_{1}\right]^{-1} \\
& (19) \quad=-\frac{D_{1}}{\lambda^{2}}\left[\mathbb{1}+\sum_{j=2}^{\frac{n-3}{2}} \lambda^{2 j-2} S_{1} C_{2 j} S_{1} D_{1} \mp i \lambda^{n-4} S_{1} v G_{n-2} v S_{1} D_{1}+\lambda^{-2} S_{1} \widetilde{M}_{0}^{ \pm}(\lambda) S_{1} D_{1}\right]^{-1} .
\end{aligned}
$$

We again only concern ourselves with explicitly finding the operators for the first two odd powers of $\lambda$ that occur. This again follows by a careful analysis of the various terms that arise in the Neumann series expansion. It is clear from the expansion (19) that the error terms for $B^{-1}(\lambda)$ are of size $\lambda^{-4} \widetilde{M}_{j}(\lambda)$ by performing the Neumann series expansion.

Since the odd powers of $\lambda$ in this expansion can only arise from odd powers in the expansion for $B^{ \pm}(\lambda)$ in combination with an even power of $\lambda$ in the Neumann series we can identify these operators explicitly. 
We note that the $\lambda^{n-6}$ term in the expansion can come only from the $\lambda^{n-2}$ term in the expansion in Lemma 2.8, In the ' $x$ ' term of the Neumann series we see

$$
-\frac{D_{1}}{\lambda^{2}}\left(\lambda^{-2}\right)\left(\mp i \lambda^{n-4} S_{1} v G_{n-2} v S_{1} D_{1}\right)= \pm i \lambda^{n-6} D_{1} v G_{n-2} v D_{1}
$$

The next odd power of $\lambda$ is the $\lambda^{n-4}$. This picks up contributions from the ' $x$ ' term in the Neumann series from the contribution of the $\lambda^{n}$ power in the expansion for $\widetilde{M}_{0}^{ \pm}(\lambda)$ in Lemma 2.8,

$$
\begin{aligned}
-\frac{D_{1}}{\lambda^{2}} & \left.\mp i \lambda^{n-2} S_{1} C_{n} D_{1}\right) \\
& = \pm i \lambda^{n-4} D_{1}\left[D_{0} v G_{n-2} v D_{0} v G_{2} v D_{0}+D_{0} v G_{2} v D_{0} v G_{n-2} v D_{0}-D_{0} v G_{n} v D_{0}\right] D_{1} \\
& = \pm i \lambda^{n-4}\left[D_{1} v G_{n-2} v D_{0} v G_{2} v D_{1}+D_{1} v G_{2} v D_{0} v G_{n-2} v D_{1}-D_{1} v G_{n} v D_{1}\right] .
\end{aligned}
$$

The other contribution is from the ' $x^{2}$ term of the Neumann series, specifically the interaction of the $\lambda^{2} S_{1} C_{4} D_{1}$ term in (19) and the $\lambda^{n-2}$ term from Lemma 2.8. These contribute

$$
\begin{gathered}
-\frac{D_{1}}{\lambda^{2}}\left\{\left(\lambda^{2} S_{1} C_{4} D_{1}\right)\left(\lambda^{n-4} S_{1} v G_{n-2} v D_{1}\right)+\left(\lambda^{n-4} S_{1} v G_{n-2} v D_{1}\right)\left(\lambda^{2} S_{1} C_{4} D_{1}\right)\right\} \\
=-\lambda^{n-4}\left(D_{1} C_{4} D_{1} v G_{n-2} v D_{1}+D_{1} v G_{n-2} v D_{1} C_{4} D_{1}\right) .
\end{gathered}
$$

Remark 2.11. The error estimates here can be more compactly summarized as

$$
B_{0}^{ \pm}(\lambda)=\widetilde{O}_{\frac{n-1}{2}}\left(\lambda^{n-6+\ell}\right), \quad B_{1}^{ \pm}(\lambda)=\widetilde{O}_{\frac{n+1}{2}}\left(\lambda^{n-4+\ell}\right), \quad B_{2}^{ \pm}(\lambda)=\widetilde{O}_{\frac{n+1}{2}}\left(\lambda^{n-2+\ell}\right),
$$

as absolutely bounded operators on $L^{2}\left(\mathbb{R}^{n}\right)$, for $0<\lambda<\lambda_{1}$.

Later on, inspired by Remark 8.3 in [16] we consider eigenfunctions with certain orthogonality conditions. Accordingly, we consider two additional cases: first when $P_{e} V G_{n-2}=$ $c_{n-2} P_{e} V 1=0$, and secondly when $P_{e} V 1=0$ and $P_{e} V x=0$ which, we see in the next Corollary, implies that $P_{e} V G_{n} V P_{e}=0$.

Corollary 2.12. Under the hypotheses of Lemma [2.10, if $P_{e} V 1=0$ then the first odd power of $\lambda$ in the expansion occurs at $\lambda^{n-4}$. That is, if $\beta>\frac{n}{2}+2+\ell$

$$
B_{ \pm}(\lambda)^{-1}=-\frac{D_{1}}{\lambda^{2}}+\sum_{j=2}^{\frac{n-3}{2}} \lambda^{2 j-4} B_{2 j}+\lambda^{n-5} B_{n-1} \pm i \lambda^{n-4} B_{n}+\widetilde{B}_{1}^{ \pm}(\lambda)
$$


If, in addition, $P_{e} V x=0$ then $B_{n}=0$ in the expansion for $B_{ \pm}(\lambda)^{-1}$. That is, if $\beta>\frac{n}{2}+4+\ell$

$$
B_{ \pm}(\lambda)^{-1}=-\frac{D_{1}}{\lambda^{2}}+\sum_{j=2}^{\frac{n-3}{2}} \lambda^{2 j-4} B_{2 j}+\lambda^{n-5} B_{n-1}+\lambda^{n-3} B_{n+1} \pm i \lambda^{n-2} B_{n+2}+\widetilde{B}_{2}^{ \pm}(\lambda) .
$$

Proof. The first claim follows clearly from Lemma 2.10 since the coefficient of $\lambda^{n-6}$ is a scalar multiple of the operator $P_{e} V 1$. This is seen through the identities,

$$
S_{1}=-S_{1} v G_{0} w=-w G_{0} v S_{1}
$$

Along with Lemma 5.5 and the fact that $D_{1}=S_{1} D_{1} S_{1}$ we see that

$$
D_{1}=S_{1} D_{1} S_{1}=w G_{0} v S_{1} D_{1} S_{1} v G_{0} w=w P_{e} w
$$

with $P_{e}$ the projection onto the eigenspace at zero. Then

$$
D_{1} v G_{n-2} v D_{1}=w P_{e} V G_{n-2} V P_{e} w=0 .
$$

We recall that $G_{n-2}=c_{n-2} 1$ in the last step.

The second claim follows from the above observations, the form of $B_{n}$ found in Lemma 2.10 and

$$
\begin{aligned}
c_{n}^{-1} D_{1} v G_{n} v D_{1} & =w P_{e} V\left(|x|^{2}-2 x \cdot y+|y|^{2}\right) V P_{e} w \\
& =w P_{e} V|x|^{2} 1 V P_{e} w-2 w P_{e} V x \cdot y V P_{e} w+w P_{e} V 1|y|^{2} V P_{e} w .
\end{aligned}
$$

We can now state several versions the expansions for $M^{ \pm}(\lambda)^{-1}$. These different expansions allow us to have finer control on the time decay rate of the error terms of the evolution given in Theorem 1.2 at the cost of more decay on the potential.

Proposition 2.13. If $|V(x)| \lesssim\langle x\rangle^{-n-8-}$, then

$$
\begin{aligned}
M^{ \pm}(\lambda)^{-1} & =-\frac{D_{1}}{\lambda^{2}}+\sum_{j=0}^{\frac{n-7}{2}} \lambda^{2 j} M_{2 j} \pm i \lambda^{n-6} D_{1} v G_{n-2} v D_{1}+\lambda^{n-5} M_{n-5} \pm i \lambda^{n-4} M_{n-4} \\
& +\lambda^{n-3} M_{n-3} \pm i \lambda^{n-2} M_{n-2}+\widetilde{O}_{\frac{n+1}{2}}\left(\lambda^{n-2+}\right)
\end{aligned}
$$

with the operators $M_{k}$ all real-valued and absolutely bounded, provided that $\lambda$ is sufficiently small.

Proof. This follows from the expansions in Lemmas 2.8 and 2.10, in the inversion lemma, Lemma 2.3. 
This expansion can be truncated, saving required decay on the potential. In particular, this longer expansion can be used to prove Corollary 1.3. We note the following useful and immediate corollaries.

Corollary 2.14. If $|V(x)| \lesssim\langle x\rangle^{-n-}$, then

$$
M^{ \pm}(\lambda)^{-1}=-\frac{D_{1}}{\lambda^{2}}+\sum_{j=0}^{\frac{n-7}{2}} \lambda^{2 j} M_{2 j} \pm i \lambda^{n-6} D_{1} v G_{n-2} v D_{1}+\widetilde{O}_{\frac{n-1}{2}}\left(\lambda^{n-6+}\right) .
$$

If $|V(x)| \lesssim\langle x\rangle^{-n-4-}$, then

$$
M^{ \pm}(\lambda)^{-1}=-\frac{D_{1}}{\lambda^{2}}+\sum_{j=0}^{\frac{n-7}{2}} \lambda^{2 j} M_{2 j} \pm i \lambda^{n-6} D_{1} v G_{n-2} v D_{1}+\lambda^{n-5} M_{n-5}+\widetilde{O}_{\frac{n-1}{2}}\left(\lambda^{n-4}\right) .
$$

Proof. The first claim follows from the expansions in Lemmas 2.8 and 2.10, in the inversion lemma, Lemma 2.3, going out to the error terms $M_{0}^{ \pm}(\lambda)$ and $B_{0}^{ \pm}(\lambda)$ respectively using $\ell=0+$.

The second claim follows from going further in the expansions in Lemmas 2.8 and 2.10. reaching $M_{1}^{ \pm}(\lambda)$ and $B_{1}^{ \pm}(\lambda)$ respectively and taking $\ell=0$.

Corollary 2.15. If $P_{e} V 1=0$ and $|V(x)| \lesssim\langle x\rangle^{-n-4-}$, then

$$
M^{ \pm}(\lambda)^{-1}=-\frac{D_{1}}{\lambda^{2}}+\sum_{j=0}^{\frac{n-7}{2}} \lambda^{2 j} M_{2 j}+\lambda^{n-5} M_{n-5} \pm i \lambda^{n-4} M_{n-4}+\widetilde{O}_{\frac{n-1}{2}}\left(\lambda^{n-4+}\right) .
$$

If $|V(x)| \lesssim\langle x\rangle^{-n-8-}$, then

$$
\begin{aligned}
M^{ \pm}(\lambda)^{-1} & =-\frac{D_{1}}{\lambda^{2}}+\sum_{j=0}^{\frac{n-7}{2}} \lambda^{2 j} M_{2 j}+\lambda^{n-5} M_{n-5} \pm i \lambda^{n-4} M_{n-4}+\lambda^{n-3} M_{n-3} \\
& \pm i \lambda^{n-2} M_{n-2}+\widetilde{O}_{\frac{n+1}{2}}\left(\lambda^{n-2+}\right)
\end{aligned}
$$

If in addition, $P_{e} V x=0$, and $|V(x)| \lesssim\langle x\rangle^{-n-8-}$, then

$$
\begin{aligned}
M^{ \pm}(\lambda)^{-1} & =-\frac{D_{1}}{\lambda^{2}}+\sum_{j=0}^{\frac{n-7}{2}} \lambda^{2 j} M_{2 j}+\lambda^{n-5} M_{n-5}+\lambda^{n-3} M_{n-3} \\
& \pm i \lambda^{n-2} M_{n-2}+\widetilde{O}_{\frac{n+1}{2}}\left(\lambda^{n-2+}\right) .
\end{aligned}
$$




\section{Dispersive estimates: the Leading terms}

In this section we use the expansions developed for $M^{ \pm}(\lambda)^{-1}$ in Section 2 to develop small $\lambda$ expansions for the operators $R_{V}^{ \pm}\left(\lambda^{2}\right)$ to determine the time decay rate of the evolution $e^{i t H} P_{a c}(H)$. We divide our analysis into three cases that depend on the eigenfunction cancellation. Specifically, we need to consider when $P_{e} V 1 \neq 0$, then when $P_{e} V 1=0$ but $P_{e} V x \neq 0$, and finally we consider when both $P_{e} V 1=0$ and $P_{e} V x=0$.

Iterating the standard resolvent identity

$$
R_{V}^{ \pm}\left(\lambda^{2}\right)=R_{0}^{ \pm}\left(\lambda^{2}\right)-R_{0}^{ \pm}\left(\lambda^{2}\right) V R_{V}^{ \pm}\left(\lambda^{2}\right)=R_{0}^{ \pm}\left(\lambda^{2}\right)-R_{V}^{ \pm}\left(\lambda^{2}\right) V R_{0}^{ \pm}\left(\lambda^{2}\right)
$$

we have the identity

$$
\begin{aligned}
R_{V}^{ \pm}\left(\lambda^{2}\right) & =\sum_{k=0}^{2 m+1}(-1)^{k} R_{0}^{ \pm}\left(\lambda^{2}\right)\left[V R_{0}^{ \pm}\left(\lambda^{2}\right)\right]^{k} \\
& +\left[R_{0}^{ \pm}\left(\lambda^{2}\right) V\right]^{m} R_{0}^{ \pm}\left(\lambda^{2}\right) v M^{ \pm}(\lambda)^{-1} v R_{0}^{ \pm}\left(\lambda^{2}\right)\left[V R_{0}^{ \pm}\left(\lambda^{2}\right)\right]^{m}
\end{aligned}
$$

In light of Lemma 2.2 the identity holds for $m+1 \geq \frac{n-3}{4}$ and $|V(x)| \lesssim\langle x\rangle^{-\frac{n+1}{2}-}$ as an identity from $L^{2, \frac{1}{2}+} \rightarrow L^{2,-\frac{1}{2}-}$, as in the limiting absorption principle.

The most singular $\lambda$ terms of the expansion, and hence the slowest decaying in time terms occur in the last term involving the operator $M^{ \pm}(\lambda)^{-1}$. These are the terms whose time decay rate is sensitive to the existence of zero-energy eigenvalues. In this section we prove the dispersive bounds for tail of the Born series. Accordingly, we focus on the contribution of (27) and put the dispersive bounds for the finite Born series terms, (26), in Section 4. In particular, we note that Proposition 4.1 ensures these terms contribute $|t|^{-\frac{n}{2}}$ as an operator from $L^{1}$ to $L^{\infty}$ to the evolution of $e^{i t H} P_{a c}(H)$.

From the '+/-' cancellation, we need only concern ourselves with the odd powers of $\lambda$ that arise in the expansion of

$$
\begin{aligned}
\left(R_{0}^{+}\left(\lambda^{2}\right) V\right)^{m} R_{0}^{+}\left(\lambda^{2}\right) v M^{+}(\lambda)^{-1} v R_{0}^{+}\left(\lambda^{2}\right)\left(V R_{0}^{+}\left(\lambda^{2}\right)\right)^{m} & \\
& -\left(R_{0}^{-}\left(\lambda^{2}\right) V\right)^{m} R_{0}^{-}\left(\lambda^{2}\right) v M^{-}(\lambda)^{-1} v R_{0}^{-}\left(\lambda^{2}\right)\left(V R_{0}^{-}\left(\lambda^{2}\right)\right)^{m}
\end{aligned}
$$

Using the algebraic fact,

$$
\prod_{k=0}^{M} A_{k}^{+}-\prod_{k=0}^{M} A_{k}^{-}=\sum_{\ell=0}^{M}\left(\prod_{k=0}^{\ell-1} A_{k}^{-}\right)\left(A_{\ell}^{+}-A_{\ell}^{-}\right)\left(\prod_{k=\ell+1}^{M} A_{k}^{+}\right),
$$

We have two cases to consider, either the '+/-' difference in (29) acts on a resolvent or on the operators $M^{ \pm}(\lambda)^{-1}$. 
3.1. No cancellation. We first consider the case in which there is no eigenfunction cancellation, that is when the operator $P_{e} V 1 \neq 0$. In this case,

Lemma 3.1. If $P_{e} V 1 \neq 0$ and $|V(x)| \lesssim\langle x\rangle^{-n-4-}$, then

$$
\text { (28) }=c_{n-2} \lambda^{n-6} P_{e} V 1 V P_{e}+\frac{E_{0}^{+}(\lambda)-E_{0}^{-}(\lambda)}{\lambda^{2}} V P_{e}+P_{e} V \frac{E_{0}^{+}(\lambda)-E_{0}^{-}(\lambda)}{\lambda^{2}}+\widetilde{O}_{\frac{n-1}{2}}\left(\lambda^{n-4}\right),
$$

which contributes $C_{n}|t|^{2-\frac{n}{2}} P_{e} V 1 V P_{e}+O\left(|t|^{1-\frac{n}{2}}\right)$ to (3) .

Proof. We first consider when the ' $+/$ ' ' difference acts on free resolvent operators $R_{0}^{ \pm}$. For the other resolvents, by Lemma 2.1, we can write

$$
R_{0}^{ \pm}\left(\lambda^{2}\right)=G_{0}+\widetilde{O}_{\frac{n-1}{2}}\left(\lambda^{2}\right)
$$

and by Corollary 2.14 ,

$$
M^{ \pm}(\lambda)^{-1}=-\frac{D_{1}}{\lambda^{2}}+\widetilde{O}_{\frac{n-1}{2}}(1) .
$$

Recall that the error terms here are to indicate that differentiation in $\lambda$ is comparable to division by $\lambda$, up to order $\frac{n-1}{2}$.

For the purposes of making sure that the $O\left(|t|^{1-\frac{n}{2}}\right)$ remainder maps between unweighted spaces, the most delicate term will be of the form

$$
\left[R_{0}^{+}\left(\lambda^{2}\right)-R_{0}^{-}\left(\lambda^{2}\right)\right]\left\{\left(V G_{0}\right)^{m} v \frac{D_{1}}{\lambda^{2}} v\left(G_{0} V\right)^{m} G_{0}+\widetilde{O}_{\frac{n-1}{2}}(1)\right\}
$$

This occurs when the '+/-' difference in (28) affects the leading free resolvents and using the expansions above for the remaining resolvents and $M^{ \pm}(\lambda)^{-1}$.

Then using the identity $S_{1}=-S_{1} v G_{0} w=-w G_{0} v S_{1}$, we have $G_{0} V G_{0} v D_{1}=$ $G_{0} v w G_{0} v S_{1} D_{1}=-G_{0} v D_{1}$ and similarly $D_{1} v G_{0} V G_{0}=-D_{1} v G_{0}$.

$$
V\left(G_{0} V\right)^{m-1} G_{0} v D_{1} v G_{0}\left(V G_{0}\right)^{m}=-V G_{0} v D_{1} v G_{0}=-V P_{e} .
$$

Where we used the definition of $P_{e}$, (52), in the last step. Now, using the expansion for $R_{0}^{ \pm}\left(\lambda^{2}\right)$ in Lemma 2.1 with $\ell=0$, we need only bound the contribution of

$$
\frac{E_{0}^{ \pm}(\lambda)}{\lambda^{2}} V P_{e}+\widetilde{O}_{\frac{n-1}{2}}\left(\lambda^{n-2}\right)
$$

By Lemma 2.1 with $\ell=0, E_{0}^{ \pm}(\lambda)=\widetilde{O}_{\frac{n-1}{2}}\left(\lambda^{n-2}\right)$, thus the first term is of size $\widetilde{O}_{\frac{n-1}{2}}\left(\lambda^{n-4}\right)$, which by Lemma 6.4, we can bound the contribution of the above term to (3) by $|t|^{1-\frac{n}{2}}$. The error term is an operator from $L^{1} \rightarrow L^{\infty}$ since we can bound it by a constant independent of $x$ and $y$, with the intermediate $z_{j}$ integrals are controlled by Lemma 2.2 and the fact that the terms in the expansion of $M^{ \pm}(\lambda)^{-1}$ are absolutely bounded on $L^{2}$. The first term can similarly be seen to map $L^{1}$ to $L^{\infty}$ since the decay of $V$ and the bounds on $E_{0}^{ \pm}$in 
Lemma 2.1 ensure that $V E_{0}^{ \pm}(\lambda)$ is in $L^{1}$, then $P_{e}: L^{1} \rightarrow L^{\infty}$ by Corollary [5.6, There is, of course, a similar term where the difference of resolvents occurs at the lagging resolvent, this contributes terms of the form

$$
P_{e} V \frac{E_{0}^{ \pm}(\lambda)}{\lambda^{2}}+\widetilde{O}_{\frac{n-1}{2}}\left(\lambda^{n-2}\right)
$$

which are controlled similarly.

The other terms in which the difference of resolvents occurs with 'inner resolvents' are similar. In fact, we note that if we have the difference on an 'inner resolvent' as in

$$
\begin{aligned}
\left(R_{0}^{-} V\right)^{j} & {\left[R_{0}^{+}\left(\lambda^{2}\right)-R_{0}^{-}\left(\lambda^{2}\right)\right]\left(V R_{0}^{+}\right)^{m-j} v \frac{D_{1}}{\lambda^{2}} v\left(R_{0}^{+} V\right)^{m} R_{0}^{+} } \\
& =\left(G_{0} V\right)^{j}\left[2 i \lambda^{n-4} G_{n-2}+E_{1}^{+}(\lambda)-E_{1}^{-}(\lambda)\right]\left(V G_{0}\right)^{m-j} v D_{1} v\left(G_{0} V\right)^{m} G_{0}+\widetilde{O}_{\frac{n-1}{2}}\left(\lambda^{n-2}\right),
\end{aligned}
$$

where we used Lemma 2.1. From the bounds on the error terms $E_{1}^{ \pm}(\lambda)$ we can integrate by parts safely as the $\left|z_{j}-z_{j+1}\right|$ powers can be absorbed by the decay of the potentials that are on either side.

The most singular term with respect to $\lambda$ occurs from the first odd power of $\lambda$ that appears in the expansion of $M^{ \pm}(\lambda)$. In this case, we need to bound

$$
\left(R_{0}^{-}\left(\lambda^{2}\right) V\right)^{m} R_{0}^{-}\left(\lambda^{2}\right) v\left[M^{+}(\lambda)^{-1}-M^{-}(\lambda)^{-1}\right] v R_{0}^{+}\left(\lambda^{2}\right)\left(V R_{0}^{+}\left(\lambda^{2}\right)\right)^{m} .
$$

By Lemma 2.1 and Corollary 2.14 this contributes

$$
\lambda^{n-6}\left(G_{0} V\right)^{m} G_{0} v D_{1} v G_{n-2} v D_{1} v G_{0}\left(V G_{0}\right)^{m}+\widetilde{O}_{\frac{n-1}{2}}\left(\lambda^{n-4}\right) .
$$

The remaining terms, which we put in the error term, are all of size $\lambda^{n-4}$ or smaller by (22) and Lemma 2.1, The operator with $\lambda^{n-6}$ can be seen to be a multiple of $P_{e} V 1 V P_{e}$ similar to the analysis for (30), we have

$$
\begin{aligned}
& \left(G_{0} V\right)^{m} G_{0} v D_{1} v G_{n-2} v D_{1} v G_{0}\left(V G_{0}\right)^{m}=G_{0} v D_{1} v G_{n-2} v D_{1} v G_{0} \\
& =G_{0} v D_{1} v G_{0} w v G_{n-2} v w G_{0} v D_{1} v G_{0}=P_{e} V G_{n-2} V P_{e}=c_{n-2} P_{e} V 1 V P_{e} .
\end{aligned}
$$

Where we used (52) in the second to last equality. By Lemma 6.3 we can bound the contribution of this to the Stone formula (3) with $C_{n}|t|^{2-\frac{n}{2}} P_{e} V 1 V P_{e}+O\left(|t|^{1-\frac{n}{2}}\right)$.

The remaining terms in the Born series, i.e. those derived from (26), have more rapid decay for large $|t|$ by Proposition 4.1, In fact using the identities for $S_{1}$ and Lemma 5.5, at this point we can write

$$
e^{i t H} P_{a c}(H)=C_{n}|t|^{2-\frac{n}{2}} P_{e} V 1 V P_{e}+O\left(|t|^{1-\frac{n}{2}}\right),
$$


where the operator $P_{e} V 1 V P_{e}$ is rank one. The weaker claim, with error term of size $o\left(|t|^{2-\frac{n}{2}}\right)$ follows by using (21) in place of (22) to obtain error terms of size $\widetilde{O}_{\frac{n-1}{2}}\left(\lambda^{n-6+}\right)$.

3.2. The case of $P_{e} V 1=0$. In Theorem 1.2, we have that if the operator $P_{e} V 1=0$, one can achieve faster time decay. In particular,

Lemma 3.2. If $P_{e} V 1=0$ and $|V(x)| \lesssim\langle x\rangle^{-n-8-}$, then

$$
(\underline{28})=\lambda^{n-4} \Gamma_{1}+\frac{E_{1}^{+}(\lambda)-E_{1}^{-}(\lambda)}{\lambda^{2}} V P_{e}+P_{e} V \frac{E_{1}^{+}(\lambda)-E_{1}^{-}(\lambda)}{\lambda^{2}}+\langle x\rangle\langle y\rangle \widetilde{O}_{\frac{n+1}{2}}\left(\lambda^{n-2}\right),
$$

with $\Gamma_{1}: L^{1} \rightarrow L^{\infty}$. This contributes $|t|^{1-\frac{n}{2}}+\langle x\rangle\langle y\rangle O\left(|t|^{-\frac{n}{2}}\right)$ to (3) .

We note that the terms with $\lambda^{-2} E_{1}^{ \pm}(\lambda)$ generically demand polynomial weights of power two in the spatial variables $x$ and $y$. These weights appear, for example, in the properties of the operator $A_{0}(t)$ in (2). We prove the more elementary generic bounds here and postpone a full account of these terms until Lemmas 3.4 and 3.6 below. In a similar vein, the error term contribution is clearly bounded by $\langle x\rangle\langle y\rangle|t|^{-\frac{n}{2}}$ via Lemma 6.4. However Lemma 4.4 will demonstrate how to bound terms of this type by $|t|^{-\frac{n}{2}}$ without weights, by shifting the stationary phase point if all $\frac{n+1}{2}$ derivatives fall on the leading (or lagging) resolvent. The sharper error estimate is not crucial here but it will be needed momentarily.

Proof. In this case, the '+/-' cancellation on $M^{ \pm}(\lambda)^{-1}$ yields a first term of size $\lambda^{n-4}$. Specifically, from Corollary 2.15 using (24), we have

$$
M^{+}(\lambda)^{-1}-M^{-}(\lambda)^{-1}=2 i \lambda^{n-4} M_{n-4}+2 i \lambda^{n-2} M_{n-2}+\widetilde{O}_{\frac{n+1}{2}}\left(\lambda^{n-2+}\right)
$$

As in the previous case, we can use Lemma 2.1 to write $R_{0}^{ \pm}\left(\lambda^{2}\right)=G_{0}+\lambda^{2} G_{2}+|x-\cdot| \widetilde{O}_{\frac{n+1}{2}}\left(\lambda^{4}\right)$, and the most singular expression in $\lambda$ occurs when the free resolvent is approximated by $G_{0}$ in each instance. Thus we have

$$
\begin{aligned}
& \left(R_{0}^{-}\left(\lambda^{2}\right) V\right)^{m} R_{0}^{-}\left(\lambda^{2}\right) v\left[M^{+}(\lambda)^{-1}-M^{-}(\lambda)^{-1}\right] v R_{0}^{+}\left(\lambda^{2}\right)\left(V R_{0}^{-}\left(\lambda^{2}\right)\right)^{m} \\
& \quad=\lambda^{n-4}\left(G_{0} V\right)^{m} G_{0} v M_{n-4} v G_{0}\left(V G_{0}\right)^{m}+\lambda^{n-2} \Gamma_{n-2}+\langle x\rangle\langle y\rangle \widetilde{O}_{\frac{n+1}{2}}\left(\lambda^{n-2+}\right)
\end{aligned}
$$

Where $\Gamma_{n-2}$ is the contribution of the operator $M_{n-2}$ with all $G_{0}$ s and the contribution of $M_{n-4}$ with $G_{0}$ s and exactly one $G_{2}$. This contributes $|t|^{1-\frac{n}{2}}+\langle x\rangle\langle y\rangle O\left(|t|^{-\frac{n}{2}}\right)$ to the Stone formula, (3), by Lemmas 6.3 and 6.4 respectively.

On the other hand, if the ' $+/-$ ' cancellation affects an outer resolvent we have to consider the contribution of terms of the form

$$
\left[R_{0}^{+}\left(\lambda^{2}\right)-R_{0}^{-}\left(\lambda^{2}\right)\right]\left(V R_{0}^{+}\left(\lambda^{2}\right)\right)^{m} v \frac{D_{1}}{\lambda^{2}} v\left(R_{0}^{+}\left(\lambda^{2}\right) V\right)^{m} R_{0}^{+}\left(\lambda^{2}\right)
$$




$$
=\left(2 i \lambda^{n-2} G_{n-2}+E_{1}^{+}(\lambda)-E_{1}^{-}(\lambda)\right)\left(V G_{0}\right)^{m} v \frac{D_{1}}{\lambda^{2}} v\left(G_{0} V\right)^{m} G_{0}+\langle x\rangle\langle y\rangle \widetilde{O}_{\frac{n+1}{2}}\left(\lambda^{n-2}\right) .
$$

We note that for the remaining terms, we use Corollary 2.15 to write $M^{ \pm}(\lambda)^{-1}=-D_{1} / \lambda^{2}+$ $M_{0}+\widetilde{O}_{\frac{n+1}{2}}\left(\lambda^{2}\right)$. Noting that $\widetilde{O}_{k}\left(\lambda^{j}\right)=\widetilde{O}_{k}\left(\lambda^{\ell}\right)$ for $\ell \leq j$, we can bound all of the terms as we do the first most singular one. The first term with the operator $G_{n-2}$ vanishes since $P_{e} V 1=0$. Thus, we are left to bound

$$
\frac{E_{1}^{ \pm}(\lambda)}{\lambda^{2}} V P_{e}
$$

Using the bounds in Lemma 2.1, this is of size $|x-\cdot|^{1+\ell} \widetilde{O}_{\frac{n+1}{2}}\left(\lambda^{n-3+\ell}\right)$. Taking $\ell=1$ in Lemma 6.4 shows that the contribution of this term is of size $\langle x\rangle^{2}|t|^{-\frac{n}{2}}$ for large $|t|$. As in Lemma 3.1, terms where the $+/$ - difference acts on an inner resolvent it can be bounded similarly. Moreover the factor of $\left|z_{j}-z_{j+1}\right|^{2}$ in the bound for $E_{1}^{ \pm}(\lambda)$ is then negated by multiplying by the potential on both sides.

There are only a finite number of terms in the expansion of finite rank operators that contribute $\lambda^{n-4}$ to the expansion. By Lemma 6.3, these contribute $|t|^{1-\frac{n}{2}}$ to the Stone formula.

The remaining claims in the second part of Theorem 1.2 come from truncating the expansion for $R_{0}^{ \pm}\left(\lambda^{2}\right)$ at $E_{0}^{ \pm}(\lambda)$ with $\ell=0$ and $\ell=0+$ respectively.

Hence we have if $P_{e} V 1=0$

$$
e^{i t H} P_{a c}(H)=|t|^{1-\frac{n}{2}} \Gamma+O\left(|t|^{-\frac{n}{2}}\right)
$$

where $\Gamma$ is a finite rank operator mapping $L^{1}$ to $L^{\infty}$, which we do not make explicit and the error term is understood as an operator between weighted spaces. Combining this with the analysis for when $P_{e} V 1 \neq 0$, we have the expansion

$$
e^{i t H} P_{a c}(H)=C_{n}|t|^{2-\frac{n}{2}} P_{e} V 1 V P_{e}+|t|^{1-\frac{n}{2}} \Gamma+O\left(|t|^{-\frac{n}{2}}\right),
$$

which is valid whether or not $P_{e} V 1=0$.

3.3. The case of $P_{e} V 1=0$ and $P_{e} V x=0$. Finally we consider the case with both cancellation conditions on the eigenfunctions at zero energy. In particular, we show that

Lemma 3.3. If $P_{e} V 1=0, P_{e} V x=0$ and $|V(x)| \lesssim\langle x\rangle^{-n-8-}$, then

$$
(\underline{28)})=\frac{R_{0}^{+}(\lambda)-R_{0}^{-}(\lambda)}{\lambda^{2}} V P_{e}+P_{e} V \frac{R_{0}^{+}(\lambda)-R_{0}^{-}(\lambda)}{\lambda^{2}}+\mathcal{E}(\lambda),
$$

which contributes $|t|^{-\frac{n}{2}}$ to (3). 
It would be convenient if we could write $\mathcal{E}(\lambda)=\widetilde{O}_{\frac{n+1}{2}}\left(\lambda^{n-2}\right)$, and this is nearly true. The issue is that one cannot bound $\left(\partial_{\lambda}\right)^{\frac{n+1}{2}} E_{0}^{ \pm}(\lambda)$ as in Lemma 2.1 without introducing spatial weights for large values of $\lambda|x-y|$. So long as that is the only obstruction, such terms can still be bounded in unweighted spaces by following the model of the finite Born series, see Lemma 4.4. We claim that every term of order $O\left(\lambda^{n-2}\right)$ in the expansion of (28) can be handled in this manner, except the ones singled out for special consideration above.

Proof. The most delicate case, for the purposes of obtaining an unweighted bound, occurs when the ' + /-' difference acts on a leading or lagging free resolvent. The goal now is to come up with an unweighted dispersive bound for the expression

$$
\int_{0}^{1} e^{i t \lambda^{2}} \chi(\lambda) \lambda^{-1}\left(R_{0}^{+}\left(\lambda^{2}\right)-R_{0}^{-}\left(\lambda^{2}\right)\right)\left(x, z_{1}\right) V\left(z_{1}\right) P_{e}\left(z_{1}, y\right) d \lambda
$$

where $P_{e}$ is the finite rank projection onto the nullspace of $-\Delta+V$. In Lemma 3.2, we showed that this term can be bounded by $\langle x\rangle^{2}|t|^{-\frac{n}{2}}$, here we wish to remove the dependence on $x$, and respectively the dependence on $y$ for the corresponding term involving $P_{e}\left(x, z_{1}\right) V\left(z_{1}\right)\left(R_{0}^{+}\left(\lambda^{2}\right)-R_{0}^{-}\left(\lambda^{2}\right)\right)\left(z_{1}, y\right)$. For clarity, we are quite explicit about the spatial variable dependence of the various operators. In particular, we show that the dependence on $x$ or $y$ in the result bounds can be changed into growth in the inner spatial variable $z_{1}$, which can be controlled by the decay of the potential $V\left(z_{1}\right)$.

In odd dimensions, one can write out

$$
R_{0}^{+}\left(\lambda^{2}\right)\left(x, z_{1}\right)=\sum_{j=0}^{\frac{n-3}{2}} \lambda^{2 j} G_{2 j}+i \lambda^{n-2} G_{n-2}+\lambda^{n-1} G_{n-1}+\lambda^{n-2} K_{+}\left(\lambda\left|x-z_{1}\right|\right),
$$

see Lemma 2.1 above. As usual $R_{0}^{-}\left(\lambda^{2}\right)$ is the complex conjugate of $R_{0}^{+}\left(\lambda^{2}\right)$.

Then $R_{0}^{+}\left(\lambda^{2}\right)-R_{0}^{-}\left(\lambda^{2}\right)=2 i \lambda^{n-2} G_{n-2}+\lambda^{n-2}\left(K_{+}\left(\lambda\left|x-z_{1}\right|\right)-K_{-}\left(\lambda\left|x-z_{1}\right|\right)\right)$ with the lower-order terms all cancelling each other. We can already write out the contribution

$$
\left(\int_{0}^{1} e^{i t \lambda^{2}} \lambda^{n-3} \chi(\lambda) d \lambda\right) G_{n-2} V P_{e}=\left(C|t|^{1-\frac{n}{2}}+O\left(|t|^{-\frac{n}{2}}\right)\right) 1 V P_{e} .
$$

These terms don't require weights, as $1 V P_{e}$ is a bounded operator from $L^{1}$ to $L^{\infty}$, this follows from the mapping properties of $P_{e}$ in Corollary 5.6 and the decay of $V$. And of course they vanish in the case where $1 V P_{e}=0$.

Estimates for the terms with $K_{ \pm}\left(\lambda\left|x-z_{1}\right|\right)$ do not require additional cancellation, so we treat only the $K_{+}$case. Near zero, i.e. when $\lambda\left|x-z_{1}\right| \leq \frac{1}{2}$, the function $K_{+}\left(\lambda\left|x-z_{1}\right|\right)$ can be viewed as a Taylor series remainder, and for large values of $\lambda\left|x-z_{1}\right|$ it follows the decay 
of the resolvent kernel, offset by the collection of monomial terms $\lambda^{j+2-n} G_{j}$. Recall that each operator $G_{j}$ has kernel $G_{j}\left(x, z_{1}\right)=c_{j}\left|x-z_{1}\right|^{2+j-n}$. Using the expansions set forth in Lemma 2.1, one sees that

$$
K_{+}(z) \sim\left\{\begin{array}{ll}
C_{n} z^{2} & \text { if } z \leq 1 \\
C_{n-1} z+C_{n-2}+\cdots \frac{C_{0}}{z^{n-2}}+C \frac{e^{i z}}{z^{\frac{n-1}{2}}} & \text { if } z>1
\end{array} .\right.
$$

And its derivatives are bounded by

$$
\left|K_{+}^{(\ell)}(z)\right| \lesssim\left\{\begin{array}{ll}
z^{2-\ell} & \text { if } z \leq 1 \\
\max \left(z^{1-\ell}, z^{\frac{1-n}{2}}\right) & \text { if } z>1
\end{array} .\right.
$$

This yields the useful uniform bound

$$
\left|K_{+}^{(\ell)}(z)\right| \lesssim z^{2-\ell}, \quad \text { for } 0 \leq \ell \leq \frac{n+3}{2},
$$

which is sufficient for most of the calculations to follow. More care is required to control $K_{+}^{(\ell)}(z)$ in a few terms that we need to differentiate to order $\ell=\frac{n+5}{2}$.

We wish to control the size of

$$
\int_{0}^{1} e^{i t \lambda^{2}} \chi(\lambda) \lambda^{n-3} K_{+}\left(\lambda\left|x-z_{1}\right|\right) d \lambda
$$

We break this into to pieces, (38) $:=T_{\text {low }}+T_{\text {med }}$, which we bound in Lemmas 3.4 and 3.6 respectively.

If the difference of free resolvents occurs affects 'inner resolvents',

$$
\left(R_{0}^{-}\left(\lambda^{2}\right) V\right)^{j}\left[R_{0}^{+}\left(\lambda^{2}\right)-R_{0}^{-}\left(\lambda^{2}\right)\right]\left(V R_{0}^{+}\left(\lambda^{2}\right)\right)^{m-j} v\left[\frac{D_{1}}{\lambda^{2}}+\widetilde{O}(1)\right]\left(R_{0}^{+} V\right)^{m} R_{0}^{+}\left(\lambda^{2}\right)
$$

the proof is somehow less delicate. The weights only occur if $\frac{n+1}{2}$ derivatives act on either the leading or lagging free resolvent. Bounding the contribution of these terms is essentially identical in form to how one controls the Born series terms in Lemma 4.4. To avoid repetition, we refer the reader to the proof of Lemma 4.4 and give only a brief sketch of the bound for these terms.

Using $R_{0}^{+}\left(\lambda^{2}\right)-R_{0}^{-}\left(\lambda^{2}\right)=\widetilde{O}_{\frac{n-1}{2}}\left(\lambda^{n-2}\right)$, we can control the $\lambda^{-2}$ singularity. Then one uses Lemmas 4.2 to see that

$$
\lambda\left(\frac{d}{d \lambda} \frac{1}{\lambda}\right)^{\frac{n-1}{2}} R_{0}^{ \pm}\left(\lambda^{2}\right)\left(x, z_{1}\right)=e^{ \pm i \lambda\left|x-z_{1}\right|} .
$$

At this point, integrating by parts against the imaginary Gaussian would result in spatial weights. So one uses a modification of stationary phase in Lemma 6.5 
Similarly, the next term in the ' + /-' difference of the operators $M^{ \pm}(\lambda)^{-1}$ is of order $\lambda^{n-2}$. That is, from Corollary 2.15 we see

$$
M^{+}(\lambda)^{-1}-M^{-}(\lambda)^{-1}=2 i \lambda^{n-2} M_{n-2}+\widetilde{O}_{\frac{n+1}{2}}\left(\lambda^{n-2+}\right) .
$$

As in the previous bounds, the contribution of this can be bounded by $|t|^{-\frac{n}{2}}$.

We now consider estimates for the low-energy contribution

$$
T_{\text {low }}=\int_{0}^{|t|^{-\frac{1}{2}}} e^{i t \lambda^{2}} \chi(\lambda) \lambda^{n-3} K_{+}\left(\lambda\left|x-z_{1}\right|\right) d \lambda .
$$

Lemma 3.4. $T_{l o w} V P_{e}$ is a bounded operator from $L^{1}$ to $L^{\infty,-2}$ whose norm is dominated by $|t|^{-\frac{n}{2}}$. If $1 V P_{e}=0$, the same is true with estimates in $L^{\infty,-1}$. If both $1 V P_{e}=0$ and $x_{j} V P_{e}=0$ for each $j \in\{1 \ldots n\}$, then the operator bound is valid between $L^{1}$ and $L^{\infty}$.

Proof. First note that $P_{e}$ is a bounded finite rank operator from $L^{1}$ to $L^{\infty}$ by Corollary 5.6. Enough decay of the potential is assumed so that Range $V P_{e} \subset L^{1,2}$, which follows if $|V(x)| \lesssim\langle x\rangle^{-n-2-}$.

It follows immediately from (37) that $\left|T_{\text {low }}\left(x, z_{1}\right)\right| \lesssim|t|^{-\frac{n}{2}}\left|x-z_{1}\right|^{2} \lesssim|t|^{-\frac{n}{2}}\langle x\rangle^{2}\left\langle z_{1}\right\rangle^{2}$, making it a bounded operator from $L^{1,2}$ to $L^{\infty,-2}$ with norm $|t|^{-n / 2}$.

In the event that $1 V P_{e}=0$, there is room for improvement due to the extra cancellation

$$
T_{\text {low }} V P_{e}=\left(\int_{0}^{|t|^{-\frac{1}{2}}} e^{i t \lambda^{2}} \lambda^{n-3}\left(K_{+}\left(\lambda\left|x-z_{1}\right|\right)-K_{+}(\lambda|x|)\right) d \lambda\right) V P_{e} .
$$

The bound on $K_{+}^{\prime}$ from (37) and the Mean Value Theorem imply that

$$
\left|K_{+}\left(\lambda\left|x-z_{1}\right|\right)-K_{+}(\lambda|x|)\right| \lesssim \lambda^{2}\left|z_{1}\right| \max \left(|x|,\left|x-z_{1}\right|\right) \lesssim \lambda^{2}\langle x\rangle\left\langle z_{1}\right\rangle^{2}
$$

which gives a bounded map from $L^{1,2}$ to $L^{\infty,-1}$ for each $\lambda>0$. The operator norm of $T_{l o w} V P_{e}$ is still controlled by $\int_{0}^{|t|^{-1 / 2}} \lambda^{n-1} d \lambda \sim|t|^{-\frac{n}{2}}$ as above.

If we further assume that the range of $V P_{e}$ is orthogonal to all linear functions, i.e. if $x V P_{e}=0$, then another layer of corrections is possible.

$$
T_{\text {low }} V P_{e}=\left(\int_{0}^{|t|^{-\frac{1}{2}}} e^{i t \lambda^{2}} \lambda^{n-3}\left(K_{+}\left(\lambda\left|x-z_{1}\right|\right)-K_{+}(\lambda|x|)+\lambda K_{+}^{\prime}(\lambda|x|) \frac{x}{|x|} \cdot z_{1}\right) d \lambda\right) V P_{e} .
$$

The Taylor remainder theorem, with respect to $x \in \mathbb{R}^{n}$, gives an upper bound

$$
\begin{aligned}
\left|K_{+}\left(\lambda\left|x-z_{1}\right|\right)-K_{+}(\lambda|x|)+\lambda K_{+}^{\prime}(\lambda|x|) \frac{x}{|x|} \cdot z_{1}\right| & \lesssim\left|z_{1}\right|^{2} \max _{z}\left(\lambda \frac{\left|K_{+}^{\prime}(\lambda z)\right|}{|z|}+\lambda^{2}\left|K_{+}^{\prime \prime}(\lambda z)\right|\right) \\
& \lesssim \lambda^{2}\left\langle z_{1}\right\rangle^{2}
\end{aligned}
$$


with the last inequality following from (37). Using the previous arguments, the range of $T_{l o w} V P_{e}$ is now bounded by $|t|^{-\frac{n}{2}}$ times a constant function in $x$, without polynomial weights.

The order of the operators $T_{l o w} V P_{e}$ occurs when the difference of free resolvents from (29) occurs on the leading resolvents. If the '+/-' difference occurs on the lagging resolvents, one must consider the contribution of $P_{e} V T_{l o w}$.

Corollary 3.5. $P_{e} V T_{\text {low }}$ is a bounded operator from $L^{1,2}$ to $L^{\infty}$ whose norm is dominated by $|t|^{-\frac{n}{2}}$. If $P_{e} V 1=0$, the same is true with estimates from $L^{1,1}$. If both $P_{e} V 1=0$ and $P_{e} V x_{j}=0$ for each $j \in\{1 \ldots n\}$, then the operator bound is valid between $L^{1}$ and $L^{\infty}$.

The same operator estimates also hold for the intermediate-energy contribution

$$
T_{m i d}=\int_{|t|^{-\frac{1}{2}}}^{1} e^{i t \lambda^{2}} \lambda^{n-3} K_{+}\left(\lambda\left|x-z_{1}\right|\right) \chi(\lambda) d \lambda .
$$

Lemma 3.6. $T_{m i d} V P_{e}$ is a bounded operator from $L^{1}$ to $L^{\infty,-2}$ whose norm is dominated by $|t|^{-\frac{n}{2}}$. If $1 V P_{e}=0$, the same is true with estimates in $L^{\infty,-1}$. If both $1 V P_{e}=0$ and $x_{j} V P_{e}=0$ for each $j \in\{1 \ldots n\}$, then the operator bound is valid between $L^{1}$ and $L^{\infty}$.

Proof. Without loss of generality, we take $t>0$. The case of $t<0$ follows similarly with obvious modifications. Integrate by parts $\frac{n-1}{2}$ times to obtain

$$
\begin{aligned}
T_{\text {mid }}\left(\left|x-z_{1}\right|\right)=t^{-\frac{n-1}{2}} \int_{t^{-\frac{1}{2}}}^{1} e^{i t \lambda^{2}}\left[\sum_{k+\ell \leq \frac{n-1}{2}} c_{k, \ell} \lambda^{k+\ell-2} F_{\ell}\left(\lambda,\left|x-z_{1}\right|\right) \chi^{(k)}(\lambda)\right] d \lambda \\
+\sum_{\ell=0}^{(n-3) / 2} c_{\ell} t^{\frac{2-n-\ell}{2}} F_{\ell}\left(t^{-\frac{1}{2}},\left|x-z_{1}\right|\right),
\end{aligned}
$$

where $F_{\ell}\left(\lambda,\left|x-z_{1}\right|\right)=\left|x-z_{1}\right|^{\ell} K_{+}^{(\ell)}\left(\lambda\left|x-z_{1}\right|\right)$. By (37), we can control

$$
\left|F_{\ell}\left(\lambda,\left|x-z_{1}\right|\right)\right| \lesssim\left|x-z_{1}\right|^{2} \lambda^{2-\ell} \quad \text { for all } \quad 0 \leq \ell \leq \frac{n+3}{2}
$$

As a result, each term in the last sum is bounded by $|t|^{-\frac{n}{2}}\left|x-z_{1}\right|^{2}$.

In the generic case, where one has some tolerance for weights, it suffices to integrate by parts again to obtain the expression

$$
T_{\text {mid }}\left(\left|x-z_{1}\right|\right)=t^{-\frac{n+1}{2}} \int_{t^{-\frac{1}{2}}}^{1} e^{i t \lambda^{2}}\left[\sum_{k+\ell \leq \frac{n+1}{2}} c_{k, \ell} \lambda^{k+\ell-4} F_{\ell}\left(\lambda,\left|x-z_{1}\right|\right) \chi^{(k)}(\lambda)\right] d \lambda
$$




$$
+\sum_{\ell=0}^{(n-1) / 2} c_{\ell} t^{\frac{2-n-\ell}{2}} F_{\ell}\left(t^{-\frac{1}{2}},\left|x-z_{1}\right|\right) .
$$

Using (40) again, the boundary term is smaller than $|t|^{-\frac{n}{2}}$, as are all the integral terms corresponding to the choice $k=0$. When $k \geq 1$, the support of $\chi^{(k)}(\lambda)$ permits a bound of $|t|^{-\frac{n+1}{2}}\left|x-z_{1}\right|^{2}$, which is an improvement for times $|t|>1$.

If $1 V P_{e}=0$, we would like to take systematic advantage of cancellation by inserting $F_{\ell}\left(\lambda,\left|x-z_{1}\right|\right)-F_{\ell}(\lambda,|x|)$ every place where $F_{\ell}\left(\lambda,\left|x-z_{1}\right|\right)$ appears in (39). In the case where all $x_{j} V P_{e}=0$ as well, the second-order remainder $F_{\ell}\left(\lambda,\left|x-z_{1}\right|\right)-F_{\ell}(\lambda,|x|)+\nabla F_{\ell}(\lambda,|x|) \cdot z_{1}$ may be used. To estimate these differences we note that for general radial functions

$$
\begin{gathered}
\left|F\left(\left|x-z_{1}\right|\right)-F(|x|)\right| \leq\left\langle z_{1}\right\rangle \max _{z}\left|F^{\prime}(z)\right| \\
\left|F\left(\left|x-z_{1}\right|\right)-F(|x|)+F^{\prime}(|x|) \frac{x}{|x|} \cdot z_{1}\right| \leq\left\langle z_{1}\right\rangle^{2} \max _{z}\left(\left|F^{\prime \prime}(z)+\frac{1}{z}\right| F^{\prime}(z) \mid\right)
\end{gathered}
$$

The maxima are taken over $z$ along the line segment joining $x$ to $x-z_{1}$, or perhaps over $|z| \leq|x|+\left|z_{1}\right| \leq 2\langle x\rangle\left\langle z_{1}\right\rangle$. As applied to the functions $F_{\ell}(\lambda, z)=|z|^{\ell} K_{+}^{(\ell)}(\lambda z)$, this yields the bounds

$$
\begin{array}{r}
\left|F_{\ell}\left(\lambda,\left|x-z_{1}\right|\right)-F_{\ell}(\lambda,|x|)\right| \lesssim \lambda^{2-\ell}\langle x\rangle\left\langle z_{1}\right\rangle^{2} \quad \text { for } 0 \leq \ell \leq \frac{n+1}{2} . \\
\left|F_{\ell}\left(\lambda,\left|x-z_{1}\right|\right)-F_{\ell}(\lambda,|x|)+\nabla F_{\ell}(\lambda,|x|) \cdot z_{1}\right| \lesssim \lambda^{2-\ell}\left\langle z_{1}\right\rangle^{2} \quad \text { for } 0 \leq \ell \leq \frac{n-1}{2} .
\end{array}
$$

The restrictions on $\ell$ come from the range of derivatives for which (37) is valid.

Suppose that $1 V P_{e}=0$. One can apply (42) to every term in (41) to obtain

$$
\left|T_{\text {mid }}\left(\left|x-z_{1}\right|\right)-T_{\text {mid }}(|x|)\right| \lesssim|t|^{-\frac{n}{2}}\langle x\rangle\langle y\rangle^{2}
$$

As $V P_{e}$ is a bounded map from $L^{1}$ to $L^{1,2}$ it follows that $\left\|\langle x\rangle^{-1} T_{m i d} V P_{e}\right\|_{L^{1} \rightarrow L^{\infty}} \lesssim|t|^{-\frac{n}{2}}$.

In the case where $1 V P_{e}=0$ and $x V P_{e}=0$, one can still apply (43) and integration by parts to every expression in (39) except for the term with $k=0$ and $\ell=\frac{n-1}{2}$. In each case the result is consistent with the expected bound

$$
\left|T_{m i d}\left(\left|x-z_{1}\right|\right)-T_{m i d}(|x|)+\nabla T_{\text {mid }}(|x|) \cdot z_{1}\right| \lesssim|t|^{-\frac{n}{2}}\left\langle z_{1}\right\rangle^{2}
$$

The remaining task is to show that the same is true for the last integral term

$$
t^{\frac{1-n}{2}} \int_{t^{-\frac{1}{2}}}^{1} e^{i t \lambda^{2}} \lambda^{\frac{n-5}{2}}\left(F_{\frac{n-1}{2}}\left(\lambda,\left|x-z_{1}\right|\right)-F_{\frac{n-1}{2}}(\lambda,|x|)+\nabla F_{\frac{n-1}{2}}(\lambda,|x|) \cdot z_{1}\right) \chi(\lambda) d \lambda
$$

The problem here is that if one integrates by parts immediately, the Taylor remainder estimate will come down to $D_{z_{1}}^{2} F_{\frac{n+1}{2}}\left(\lambda,\left|x-z_{1}\right|\right)$, and (37) is false for $z>1$ if we're taking that many derivatives. What goes wrong specifically is that the oscillatory function is really 
$e^{i t \lambda^{2}} e^{i \lambda\left|x-z_{1}\right|}$ with the second part coming from the resolvent kernel. The stationary phase point is therefore $\lambda=-\left|x-z_{1}\right| / 2 t$ instead of zero.

The dependence on $z_{1}$ is inconvenient, since $z_{1}$ is also a variable in the next operator down the line. To work around it, we adopt the technique, due to Yajima, of placing the stationary phase point at $\lambda_{0}=-|x| / 2 t$ instead. The discrepancy between $|x|$ and $\left|x-z_{1}\right|$ will cost us a factor of $\left\langle z_{1}\right\rangle$ which gets absorbed into the decay of the potential.

To set up the calculation, let $\tilde{F}(\lambda, x, y)=\lambda^{\frac{n-5}{2}} e^{-i \lambda|x|} F_{\frac{n-1}{2}}\left(\lambda,\left|x-z_{1}\right|\right)$. We will apply the general stationary phase bound from Lemma 6.5.

$\left|\int_{t^{-\frac{1}{2}}}^{1} e^{i t\left(\lambda-\lambda_{0}\right)^{2}} F\left(\lambda, x, z_{1}\right) \chi(\lambda) d \lambda\right| \lesssim|t|^{-\frac{1}{2}} \sup _{\lambda}\left|F\left(\lambda, x, z_{1}\right)\right|+|t|^{-\frac{3}{4}}\left[\int_{t^{-\frac{1}{2}}}^{1}\left|\frac{\partial F}{\partial \lambda}\left(\lambda, x, z_{1}\right)\right|^{2} d \lambda\right]^{\frac{1}{2}}$.

In particular we need to apply Lemma 6.5 to the functions $D_{z_{1}}^{2} \tilde{F}(\lambda, x, z)$ and determine what bounds hold uniformly over $x \in \mathbb{R}^{n}$ and $|z| \leq\left|z_{1}\right|$.

The supremum estimate for $D_{z_{1}}^{2} \tilde{F}$ is essentially the same as (43). With $z_{1 j}$ the $j^{\text {th }}$ component of $z_{1}$,

$$
\begin{aligned}
& D_{z_{1 i} z_{1 j}}^{2} \tilde{F}(\lambda, x, z)= \lambda^{\frac{n-5}{2}} e^{-i \lambda|x|} D_{z_{1 i} z_{1 j}}^{2} F_{\frac{n-1}{2}}(\lambda,|x-z|) \\
&=\frac{\left(x_{i}-z_{i}\right)\left(x_{j}-z_{j}\right) \lambda \frac{n-3}{2} e^{-i \lambda|x|}}{|x-z|^{4}}\left(\lambda F_{\frac{n+3}{2}}(\lambda,|x-z|)-2 F_{\frac{n+1}{2}}(\lambda,|x-z|)\right) \\
& \quad+\delta_{i j} \frac{\lambda^{\frac{n-3}{2}}}{|x-z|^{2}} e^{-i \lambda|x|} F_{\frac{n+1}{2}}(\lambda,|x-z|) .
\end{aligned}
$$

These expressions are all of unit size by (40), therefore

$$
\left|\tilde{F}\left(\lambda, x, z_{1}\right)-\tilde{F}(\lambda, x, 0)-\nabla_{y} \tilde{F}(\lambda, x, 0) \cdot z_{1}\right| \lesssim\left\langle z_{1}\right\rangle^{2} \text { for all } \lambda \geq 0 \text {. }
$$

Most of the estimates for $\partial_{\lambda} D_{z_{1}}^{2} \tilde{F}(\lambda, x, z)$ follow a similar nature. If the $\lambda$-derivative falls on a power of $\lambda$, the resulting expression will be controlled by $\lambda^{-1}$, and $\int_{t^{-\frac{1}{2}}}^{1} \lambda^{-2} d \lambda \leq|t|^{\frac{1}{2}}$.

For $\frac{d}{d \lambda}\left[e^{-i \lambda|x|} F_{\ell}(\lambda,|x-z|)\right]$, we use the more detailed information in (35) to conlude that

$$
e^{-i \lambda|x|} F_{\ell}(\lambda,|x-z|) \sim\left\{\begin{array}{cl}
|x-z|^{2} e^{-i \lambda|x|} \lambda^{2-\ell}, & \text { if } \lambda|x-z| \leq 1 \\
\frac{|x-z|}{\lambda^{\ell-1}} e^{-i \lambda|x|}+\frac{e^{i \lambda(|x-z|-|x|)}}{|x-z|^{\frac{n-1}{2}-\ell} \lambda^{\frac{n-1}{2}}}, & \text { if } \lambda|x-z|>1
\end{array}\right.
$$

In the regime where $\lambda|x-z| \leq 1$, this yields

$$
\frac{d}{d \lambda}\left[e^{-i \lambda|x|} F_{\ell}(\lambda,|x-z|)\right] \lesssim|x-z|^{2} \lambda^{2-\ell}\left(|z|+|x-z|+\lambda^{-1}\right) \lesssim|x-z|^{2} \lambda^{2-\ell}\left(|z|+\lambda^{-1}\right)
$$

In the regime where $\lambda|x-z|>1$, it gives

$$
\frac{d}{d \lambda}\left[e^{-i \lambda|x|} F_{\ell}(\lambda,|x-z|)\right]
$$




$$
\begin{aligned}
& \lesssim|x-z| \lambda^{1-\ell}\left(|z|+|x-z|+\lambda^{-1}\right)+\frac{|x-z|^{2} \lambda^{2-\ell}}{(\lambda|x-z|)^{\frac{n+3}{2}-\ell}}\left(\lambda^{-1}+(|x-z|-|x|)\right) \\
& \lesssim|x-z|^{2} \lambda^{2-\ell}\left(|z|+\lambda^{-1}\right)
\end{aligned}
$$

so long as $\ell \leq \frac{n+3}{2}$. The end result is that $\left|\frac{d}{d \lambda} D_{z_{1}}^{2} \tilde{F}(\lambda, x, z)\right| \lesssim|z|+\lambda^{-1}$, and consequently

$$
\left|\frac{d}{d \lambda}\left[\tilde{F}\left(\lambda, x, z_{1}\right)-\tilde{F}(\lambda, x, 0)-\nabla_{y} \tilde{F}(\lambda, x, 0) \cdot z_{1}\right]\right| \lesssim\left\langle z_{1}\right\rangle^{3}+\left\langle z_{1}\right\rangle^{2} \lambda^{-1} \text { for all } \lambda \geq 0 .
$$

Over the interval $\left[t^{-1 / 2}, 1\right]$, the $L^{2}$ norm of this function is bounded by $|t|^{\frac{1}{4}}\left\langle z_{1}\right\rangle^{3}$ for $|t| \geq 1$.

Finally, once this estimate and (44) are applied in the context of Lemma 6.5, we can conclude that

$$
\left|T_{\text {mid }}\left(\left|x-z_{1}\right|\right)-T_{\text {mid }}(|x|)+\nabla T_{\text {mid }}(|x|) \cdot y z_{1}\right| \lesssim|t|^{-\frac{n}{2}}\left\langle z_{1}\right\rangle^{3}
$$

Under the assumptions that $1 V P_{e}$ and $x_{j} V P_{e}$ all vanish, and with the decay assumptions on $V, V P_{e}$ maps into the weighted space $L^{1,3}\left(\mathbb{R}^{n}\right)$, it follows that $\left\|T_{\text {mid }} V P_{e} f\right\|_{\infty} \lesssim|t|^{-\frac{n}{2}}\|f\|_{1}$ for all $|t| \geq 1$.

As before, we have the following immediate corollary.

Corollary 3.7. $P_{e} V T_{\text {mid }}$ is a bounded operator from $L^{1,2}$ to $L^{\infty}$ whose norm is dominated by $|t|^{-\frac{n}{2}}$. If $P_{e} V 1=0$, the same is true with estimates from $L^{1,1}$. If both $P_{e} V 1=0$ and $P_{e} V x_{j}=0$ for each $j \in\{1 \ldots n\}$, then the operator bound is valid between $L^{1}$ and $L^{\infty}$.

\section{Dispersive BOUndS FOR THE FINITE Born SERIES}

In this section we consider the contribution of the finite Born series terms, (26). We prove

Proposition 4.1. The contribution of (26) to (3) is bounded by $|t|^{-\frac{n}{2}}$ uniformly in $x$ and y. That is,

$$
\sup _{x, y \in \mathbb{R}^{n}}\left|\int_{0}^{\infty} e^{i t \lambda^{2}} \lambda \chi(\lambda)\left[\sum_{k=0}^{2 m+1}(-1)^{k}\left\{R_{0}^{+}\left(V R_{0}^{+}\right)^{k}-R_{0}^{-}\left(V R_{0}^{-}\right)^{k}\right\}\right]\left(\lambda^{2}\right)(x, y) d \lambda\right| \lesssim|t|^{-\frac{n}{2}} .
$$

For the first term of the Born series, when $k=0$ in (26), we define

$$
\mathcal{G}_{1}(\lambda, r)=C_{1} \frac{e^{i \lambda r}}{\lambda}
$$

We then note the identity 
Lemma 4.2. For $n \geq 3$ and odd, the following recurrence relation holds.

$$
\left(\frac{1}{\lambda} \frac{d}{d \lambda}\right) \mathcal{G}_{n}(\lambda, r)=\frac{1}{2 \pi} \mathcal{G}_{n-2}(\lambda, r) .
$$

Proof. The proof follows from the recurrence relations of the Hankel functions, found in [1] and the representation of the kernel given in (4). One can also prove this (with a fixed constant instead of $2 \pi$ ) directly using (5).

This 'dimension reduction' identity says that, up to a constant factor, the operation of $\frac{1}{\lambda} \frac{d}{d \lambda}$ takes an $n$ dimensional free resolvent to an $n-2$ dimensional free resolvent.

Lemma 4.3. We have the bound

$$
\sup _{x, y \in \mathbb{R}^{n}}\left|\int_{0}^{\infty} e^{i t \lambda^{2}} \lambda \chi(\lambda)\left[R_{0}^{+}\left(\lambda^{2}\right)-R_{0}^{-}\left(\lambda^{2}\right)\right](x, y) d \lambda\right| \lesssim|t|^{-\frac{n}{2}} .
$$

Proof. We note that, by Lemma 2.1 we can safely integrate by parts $\frac{n-1}{2}$ times without boundary terms or growth in $|x-y|$. We consider the case when all derivatives act on the resolvents. In this case, using Lemma 4.2, we have to bound

$$
\begin{aligned}
\mid \frac{1}{(2 i t)^{\frac{n-1}{2}}} \int_{0}^{\infty} e^{i t \lambda^{2}} & \chi(\lambda) \lambda\left(\frac{1}{\lambda} \frac{d}{d \lambda}\right)^{\frac{n-1}{2}}\left[R_{0}^{+}\left(\lambda^{2}\right)-R_{0}^{-}\left(\lambda^{2}\right)\right](x, y) d \lambda \mid \\
& \lesssim \frac{1}{|t|^{\frac{n-1}{2}}}\left|\int_{0}^{\infty} e^{i t \lambda^{2}} \chi(\lambda)\left(e^{i \lambda|x-y|}-e^{-i \lambda|x-y|}\right) d \lambda\right| \lesssim|t|^{-\frac{n}{2}}
\end{aligned}
$$

Here the last half power of time decay follows from Parseval and the facts that $\| \chi^{\vee}(\cdot \pm \mid x-$ $y \mid) \|_{1} \lesssim 1$ uniformly in $x$ and $y$ and $\left\|\widehat{e^{i t(\cdot)^{2}}}\right\|_{\infty} \lesssim|t|^{-\frac{1}{2}}$.

In the case in which one (or more) derivatives act on the cut-off we can integrate by parts at least $\frac{n+1}{2}$ times. The derivative bounds in Lemma 2.1 hold without any growth in $|x-y|$ since at most $\frac{n-1}{2}$ derivatives act on the error term and $\chi^{(k)}(\lambda)$ is supported on the set $\lambda \approx 1$.

Lemma 4.4. For $k \geq 1$, we have the bound

$$
\sup _{x, y \in \mathbb{R}^{n}}\left|\int_{0}^{\infty} e^{i t \lambda^{2}} \lambda \chi(\lambda)\left[\left(R_{0}^{+}\left(\lambda^{2}\right) V\right)^{k} R_{0}^{+}\left(\lambda^{2}\right)-\left(R_{0}^{-}\left(\lambda^{2}\right) V\right)^{k} R_{0}^{-}\left(\lambda^{2}\right)\right](x, y) d \lambda\right| \lesssim|t|^{-\frac{n}{2}},
$$

provided $|V(x)| \lesssim\langle x\rangle^{-\frac{n+3}{2}-}$.

Proof. We first note that, by Lemma 2.1, we have

$$
\left(R_{0}^{ \pm}\left(\lambda^{2}\right) V\right)^{k} R_{0}^{ \pm}\left(\lambda^{2}\right)=K_{0}+\lambda^{2} K_{2}+\cdots+\lambda^{n-3} K_{n-3}+E_{k}^{ \pm}(\lambda)
$$


Here $K_{j}$ are real-valued, absolutely bounded operators. One can identify these explicitly, for instance

$$
K_{0}=G_{0}\left(V G_{0}\right)^{k}, \quad K_{2}=\sum_{j=0}^{k}\left(G_{0} V\right)^{j} G_{2}\left(V G_{0}\right)^{k-j},
$$

though this is not important in our analysis. Further, $E_{k}^{ \pm}(\lambda)$ satisfy the bounds

$$
\left|\partial_{\lambda}^{j} E_{k}^{ \pm}(\lambda)\right| \lesssim \lambda^{n-2-j}, \quad j=0,1, \ldots, \frac{n-1}{2} .
$$

That is to say $E_{k}^{ \pm}(\lambda)=\widetilde{O}_{\frac{n-1}{2}}\left(\lambda^{n-2}\right)$. One can also write $E_{k}^{ \pm}(\lambda)=\langle x\rangle\langle y\rangle \widetilde{O}_{\frac{n+1}{2}}\left(\lambda^{n-2}\right)$ to see that one can attain the $|t|^{-\frac{n}{2}}$ decay rate as an operator between weighted spaces. The $\frac{n+1}{2}$ st derivative requires slightly more care to avoid spatial weights.

We need to bound

$$
\begin{aligned}
\int_{0}^{\infty} e^{i t \lambda^{2}} \lambda \chi(\lambda)\left[\left(R_{0}^{+}\left(\lambda^{2}\right) V\right)^{k} R_{0}^{+}\left(\lambda^{2}\right)-\left(R_{0}^{-}\right.\right. & \left.\left.\left(\lambda^{2}\right) V\right)^{k} R_{0}^{-}\left(\lambda^{2}\right)\right](x, y) d \lambda \\
& =\int_{0}^{\infty} e^{i t \lambda^{2}} \lambda \chi(\lambda)\left[E_{k}^{+}(\lambda)-E_{k}^{-}(\lambda)\right](x, y) d \lambda .
\end{aligned}
$$

The error bounds on $E_{k}^{ \pm}(\lambda)$ formally allow us to integrate by part $\frac{n-1}{2}$ times to bound

$$
\frac{1}{(2 i t)^{\frac{n-1}{2}}} \int_{0}^{\infty} e^{i t \lambda^{2}} \lambda\left(\frac{1}{\lambda} \frac{d}{d \lambda}\right)^{\frac{n-1}{2}} \chi(\lambda)\left[E_{k}^{+}(\lambda)-E_{k}^{-}(\lambda)\right](x, y) d \lambda .
$$

As in the free case, if at least one derivative acts on the cut-off, the unweighted bound is clear. On the other hand, if all the derivatives act on the error functions, we need only worry (about weights) if all the derivatives act on either the first (leading) resolvent $R_{0}^{ \pm}\left(\lambda^{2}\right)\left(x, z_{1}\right)$ or the last (lagging) resolvent $R_{0}^{ \pm}\left(\lambda^{2}\right)\left(z_{k}, y\right)$. Without loss of generality, we consider the first case and no longer count on the ' $+/$-' cancellation. Then, via the 'dimension reduction' identity, Lemma 4.2, we have to bound

$$
\frac{1}{(2 i t)^{\frac{n-1}{2}}} \int_{0}^{\infty} e^{i t \lambda^{2}} e^{ \pm i \lambda\left|x-z_{1}\right|} V\left(R_{0}^{ \pm}\left(\lambda^{2}\right) V\right)^{k-1} R_{0}^{ \pm}\left(\lambda^{2}\right)\left(z_{1}, y\right) d \lambda
$$

To see this, we note that the first resolvent contributes

$$
R_{0}^{ \pm}\left(\lambda^{2}\right)\left(x, z_{1}\right)-G_{0}-\lambda^{2} G_{2}-\cdots-\lambda^{n-3} G_{n-3}
$$

to $E_{k}^{ \pm}(\lambda)$. After $\frac{n-1}{2}$ integration by parts, the contribution is

$$
\begin{array}{r}
\lambda\left(\frac{1}{\lambda} \frac{d}{d \lambda}\right)^{\frac{n-1}{2}}\left[R_{0}^{ \pm}\left(\lambda^{2}\right)\left(x, z_{1}\right)-G_{0}-\lambda^{2} G_{2}-\cdots-\lambda^{n-3} G_{n-3}\right] \\
=\lambda\left(\frac{1}{\lambda} \frac{d}{d \lambda}\right)^{\frac{n-1}{2}}\left[R_{0}^{ \pm}\left(\lambda^{2}\right)\left(x, z_{1}\right)\right]=C_{1} e^{ \pm i \lambda\left|x-z_{1}\right|}
\end{array}
$$


Here we use the technique of moving the stationary point of the phase to integrate by parts another time. Without loss of generality we take $t>0$, the case of $t<0$ follows with minor adjustments. In particular, we have to extract another $t^{-\frac{1}{2}}$ from

$$
\begin{gathered}
\int_{0}^{\infty} e^{i t \lambda^{2} \mp i \lambda|x|} e^{ \pm i \lambda\left(\left|x-z_{1}\right|-|x|\right)} V\left(R_{0}^{ \pm}\left(\lambda^{2}\right) V\right)^{k-1} R_{0}^{ \pm}\left(\lambda^{2}\right)\left(z_{1}, y\right) d \lambda \\
=\int_{0}^{\infty} e^{i t \lambda^{2} \mp i \lambda|x|} e^{ \pm i \lambda\left(\left|x-z_{1}\right|-|x|\right)} \widetilde{O}_{1}(1) d \lambda
\end{gathered}
$$

Where the bounds on $V\left(R_{0}^{ \pm}\left(\lambda^{2}\right) V\right)^{k-1} R_{0}^{ \pm}\left(\lambda^{2}\right)$ follow from Lemma 2.1, We then break up the integral into two pieces, on $0<\lambda<t^{-\frac{1}{2}}$ the extra $t^{-\frac{1}{2}}$ decay is easy to see. Using Lemma 6.5 with

$$
F\left(\lambda, x, z_{1}\right)=e^{-i|x|^{2} / 4 t^{2}} e^{ \pm i \lambda\left(\left|x-z_{1}\right|-|x|\right)} \widetilde{O}_{1}(1),
$$

we can gain the extra $t^{-\frac{1}{2}}$ time decay on the remaining piece $t^{-\frac{1}{2}}<\lambda<1$. We can see that $\sup _{\lambda}\left|F\left(\lambda, x, z_{1}\right)\right| \lesssim 1$ and $\left|\partial_{\lambda} F\left(\lambda, x, z_{1}\right)\right| \lesssim\left\langle z_{1}\right\rangle+\lambda^{-1}$. So that

$$
\begin{aligned}
t^{-\frac{3}{4}}\left(\int_{t^{-\frac{1}{2}}}^{1}\left|\partial_{\lambda} F\left(\lambda, x, z_{1}\right)\right|^{2} d \lambda\right)^{\frac{1}{2}} & \lesssim t^{-\frac{3}{4}}\left(\int_{t^{-\frac{1}{2}}}^{1}\left\langle z_{1}\right\rangle^{2}+\lambda^{-2} d \lambda\right)^{\frac{1}{2}} \lesssim t^{-\frac{3}{4}}\left(\left\langle z_{1}\right\rangle^{2}+t^{\frac{1}{2}}\right)^{\frac{1}{2}} \\
& \lesssim t^{-\frac{3}{4}}\left\langle z_{1}\right\rangle+t^{-\frac{1}{2}} \lesssim t^{-\frac{1}{2}}\left\langle z_{1}\right\rangle .
\end{aligned}
$$

The growth in $z_{1}$ can be absorbed by decay of the potential $V\left(z_{1}\right)$.

The choice of decay rate on the potential is chosen to control the spatial integrals which arise in the iteration of resolvents. We note that

$$
\left|\partial_{\lambda}^{j} R_{0}^{ \pm}\left(\lambda^{2}\right)(x, y)\right| \lesssim|x-y|^{j+2-n}+\lambda^{\frac{n-3}{2}}|x-y|^{j+\frac{1-n}{2}}
$$

The bounds here are developed in the proof of Lemma 2.1. The decay required on the potential is dictated by the second term. Using this as our primary bound, the terms in the integral of kernel of $\partial_{\lambda}^{\frac{n+1}{2}}\left(R_{0}^{ \pm}\left(\lambda^{2}\right) V\right)^{k} R_{0}^{ \pm}\left(\lambda^{2}\right)$ which require the most decay from the potential are of the form

$$
\int_{\mathbb{R}^{k n}} \frac{1}{\left|x-z_{1}\right|^{\frac{n-1}{2}-\alpha_{0}}} \prod_{j=1}^{k} \frac{V\left(z_{j}\right)}{\left|z_{j}-z_{j+1}\right|^{\frac{n-1}{2}-\alpha_{j}}} d \vec{z},
$$

where $\alpha_{j} \in \mathbb{N}_{0}$ and $\sum \alpha_{j}=\frac{n+1}{2}, z_{k+1}=y$ and $d \vec{z}=d z_{1} d z_{2} \cdots d z_{k}$. (There is of course the caveat that if say $\alpha_{0}=\frac{n+1}{2}$ the last power of $\left|x-z_{1}\right|$ is really replaced with $\left\langle z_{1}\right\rangle$, and similarly if $\alpha_{k}=\frac{n+1}{2}$, the last $\left|z_{k}-y\right|$ is replaced by $\left\langle z_{k}\right\rangle$.) Arithmetic-geometric mean inequalities allow us to consider instead the integral

$$
\int_{\mathbb{R}^{k n}} \frac{1}{\left|x-z_{1}\right|^{\frac{n-1}{2}}} \prod_{j=1}^{k} \frac{V\left(z_{j}\right)}{\left|z_{j}-z_{j+1}\right|^{\frac{n-1}{2}}}\left(\left\langle z_{1}\right\rangle\left|x-z_{1}\right|^{\frac{n-1}{2}}\right.
$$




$$
\left.+\sum_{\ell=2}^{k-1}\left|z_{\ell}-z_{\ell+1}\right|^{\frac{n+1}{2}}+\left\langle z_{k}\right\rangle\left|z_{k}-y\right|^{\frac{n-1}{2}}\right) d \vec{z}
$$

as the quantity in parentheses dominates any product of $\left|z_{\ell}-z_{\ell+1}\right|$ of order $\frac{n+1}{2}$. Choose a representative element from the summation over $\ell$, this negates a factor of $\left|z_{\ell}-z_{\ell+1}\right|^{(1-n) / 2}$ in the product and replaces it with $\left|z_{\ell}-z_{\ell+1}\right| \lesssim\left\langle z_{\ell}\right\rangle\left\langle z_{\ell+1}\right\rangle$. Then we may consider

$$
\int_{\mathbb{R}^{k n}}\left(\frac{1}{\left|x-z_{1}\right|^{\frac{n-1}{2}}} \prod_{j=1}^{\ell-1} \frac{V\left(z_{j}\right)}{\left|z_{j}-z_{j+1}\right|^{\frac{n-1}{2}}}\left\langle z_{\ell}\right\rangle\right)\left(\left\langle z_{\ell+1}\right\rangle \prod_{j=\ell}^{k} \frac{V\left(z_{j}\right)}{\left|z_{j}-z_{j+1}\right|^{\frac{n-1}{2}}}\right) d \vec{z} .
$$

Assuming $|V(z)| \lesssim\langle z\rangle^{-\beta}$, the integral over $z_{\ell}$ takes the form

$$
\int_{\mathbb{R}^{n}} \frac{\left\langle z_{\ell}\right\rangle^{1-\beta}}{\left|z_{\ell-1}-z_{\ell}\right|^{\frac{n-1}{2}}} d z_{\ell}
$$

If $\beta>\frac{n+3}{2}$, using Lemma 6.1 it is clear that

$$
\sup _{z_{\ell-1} \in \mathbb{R}^{n}} \mid(\underline{48)} \mid \lesssim 1
$$

After this, the integral over $z_{\ell-1}$ can be bounded by (48), then the integral over $z_{\ell-2}$ and so on. The integrals over $z_{\ell+1}, z_{\ell+2}, \ldots, z_{k}$ are treated in an identical manner so the the entire integral with respect to $\vec{z}$ is bounded uniformly in $x$ and $y$.

If one uses the first term in the bound (47) exclusively, there are some problems with local singularities due to the fact that, for example by Corollary 6.2,

$$
\int_{\mathbb{R}^{n}} \frac{V(z)}{|x-z|^{n-2}|z-y|^{n-2}} d z \lesssim|x-y|^{4-n}
$$

may still be singular for small values of $|x-y|$. Fortunately, the local singularities are better behaved than the expression (47) would indicate due to the cancellation of the leading terms of the expansion in (45). The spatial part of the terms that contribute to $E_{k}^{ \pm}(\lambda)$ are of the form

$$
G_{0+\alpha_{0}}\left(x, z_{1}\right) \prod_{j=1}^{k} G_{0+\alpha_{j}}\left(z_{j}, z_{j+1}\right) V\left(z_{j}\right)
$$

with $\sum \alpha_{j}=n-2$ to account for the fact that $E_{k}^{ \pm}$is of order $\lambda^{n-2}$. Collectively there is an improvement of $n-2$ powers of local regularity. Where $G_{n-2}$ appears in an expression it may be represent both $G_{n-2}$ and the error term $E_{0}^{ \pm}(\lambda)$ from Lemma 2.1, which are both bounded by 1 with respect to the spatial variables. In the worst case with respect to the spatial singularities, all the $\lambda$ derivatives act on the cut-off function $\chi$ rather than on 
resolvents. Then for a fixed value of $\lambda$ we can bound their contribution by

$$
\begin{aligned}
\int_{\mathbb{R}^{k n}} \frac{1}{\left|x-z_{1}\right|^{n-2}} \prod_{j=1}^{k} \frac{V\left(z_{j}\right)}{\left|z_{j}-z_{j+1}\right|^{n-2}}\left(\left|x-z_{1}\right|^{n-2}\right. \\
\left.\quad+\sum_{\ell=2}^{k-1}\left|z_{\ell}-z_{\ell+1}\right|^{n-2}+\left|z_{k}-y\right|^{n-2}\right) d \vec{z} .
\end{aligned}
$$

The quantity in parentheses dominates any product of $\left|z_{\ell}-z_{\ell+1}\right|$ of order $n-2$. If $\beta>2$, we note that any representative term is controlled by the bound

$$
\sup _{z_{j-1} \in \mathbb{R}} \int_{\mathbb{R}^{n}} \frac{\left\langle z_{j}\right\rangle^{-\beta}}{\left|z_{j-1}-z_{j}\right|^{n-2}} d z_{j} \lesssim 1
$$

iterated $k$ times, again starting with $z_{\ell}$ and $z_{\ell+1}$. Any other integrals with respect to $\vec{z}$ can be bounded by a combination of the two cases considered.

We can now prove the main Theorem.

Proof of Theorem 1.2. We note that the Theorem is proven by bounding the oscillatory integral in the Stone formula (3),

$$
\left.\mid \int_{0}^{\infty} e^{i t \lambda^{2}} \lambda \chi(\lambda)\left[R_{V}^{+}\left(\lambda^{2}\right)-R_{V}^{-}\left(\lambda^{2}\right)\right](x, y)\right)\left.d \lambda\left|\lesssim_{x, y}\right| t\right|^{-\alpha}
$$

We begin by proving Part (11), where there is no $x, y$ dependence. The proof follows by expanding $R_{V}^{ \pm}\left(\lambda^{2}\right)$ into the Born series expansion, (26) and (27). The contribution of (26) is bounded by $|t|^{-\frac{n}{2}}$ by Proposition 4.1, while the contribution of (27) is bounded by $C_{n}|t|^{2-\frac{n}{2}} P_{e} V 1 V P_{e}+O\left(|t|^{2-\frac{n}{2}}\right)$ by Lemma 3.1

To prove Part (2), one uses Lemma 3.2 in the place of Lemma 3.1] in the proof of Part 1 Finally, Part (3) is proven by using Lemma 3.3.

We note that the proof of Theorem 1.1 is actually simpler. If zero is regular $S_{1}=0$, so many terms drop out of the expansions. In addition, the expansion of $M^{ \pm}(\lambda)^{-1}$ is of the same form with respect to the spectral variable $\lambda$ as $\left(M^{ \pm}(\lambda)+S_{1}\right)^{-1}$ given in Lemma 2.8 with different operators, see Remark 2.9. The dispersive bounds follow as in the analysis when zero is not regular. 


\section{Spectral Characterization}

We prove a characterization of the spectral subspaces of $L^{2}\left(\mathbb{R}^{n}\right)$ that are related to the invertibility of certain operators in our expansions. This characterization is essentially Lemmas 5-7 of [8] for three-dimensional Schrödinger operators modified as needed to fit higher spatial dimensions. Similar characterizations appear in Section 5 of [7] for twodimensional Schrödinger operators and Section 7 of [5] for four dimensions. The proofs here are slightly simpler, as we do not need to account for zero energy resonances. In this section it is assumed that $V$ is not identically zero.

In contrast the rest of the paper, in this section $n \geq 5$ need not be odd.

Lemma 5.1. Assume that $|V(x)| \lesssim\langle x\rangle^{-2 \beta}$ for some $\beta \geq 2, f \in S_{1} L^{2}\left(\mathbb{R}^{n}\right) \backslash\{0\}$ for $n \geq 5$ iff $f=w g$ for $g \in L^{2} \backslash\{0\}$ such that $-\Delta g+V g=0$ in $\mathcal{S}^{\prime}$.

Proof. First note that

$$
(-\Delta+V) g=0 \Leftrightarrow\left(I+G_{0} V\right) g=0 .
$$

Now suppose $f \in S_{1} L^{2} \backslash\{0\}$, that is $f \neq 0$ and $f \in \operatorname{ker}\left(U+v G_{0} v\right)$. So that

$$
0=\left(\left[U+v G_{0} v\right] f\right)(x)=U(x) f(x)+c_{0} v(x) \int_{\mathbb{R}^{n}} \frac{v(y) f(y)}{|x-y|^{n-2}} d y .
$$

Or,

$$
f(x)+c_{0} w(x) \int_{\mathbb{R}^{n}} \frac{v(y) f(y)}{|x-y|^{n-2}} d y=0 .
$$

Let

$$
g(x)=-c_{0} \int_{\mathbb{R}^{n}} \frac{v(y) f(y)}{|x-y|^{n-2}} d y=-G_{0} v f
$$

So that,

$$
g(x)=-G_{0} v f(x)=-G_{0} v(w g)(x)=-G_{0} V g(x) \quad \Rightarrow \quad\left(I+G_{0} V\right) g=0 .
$$

To see that $g \in L^{2}$, we note that $g(x)=-C_{n} I_{2}(v f)(x)$ with $I_{2}$ a Riesz potential. Noting, for instance, Lemma 2.3 of [16] we have $I_{2}: L^{2, s} \rightarrow L^{2,-s^{\prime}}$ if $s, s^{\prime} \geq 0$ and $s+s^{\prime} \geq 2$. Note that if $s=\beta \geq 2$ and $s^{\prime}=0$ we have $v f \in L^{2,2}$ and then $I_{2}(v f) \in L^{2}$.

On the other hand, assume $g \in L^{2} \backslash\{0\}$ such that $-\Delta g+V g=0$ in $\mathcal{S}^{\prime}$. Then, denoting $f=w g$, we have $f \in L^{2, \beta}$.

$$
\begin{aligned}
\left(U+v G_{0} v\right) f(x) & =U(x) f(x)+C_{n} v(x) \int_{\mathbb{R}^{n}} \frac{v(y) f(y)}{|x-y|^{n-2}} d y \\
& =U(x) w(x) g(x)+C_{n} v(x) \int_{\mathbb{R}^{n}} \frac{v(y) U(y) w(y) g(y)}{|x-y|^{n-2}} d y
\end{aligned}
$$




$$
=v(x) g(x)+v(x) G_{0} V g(x)=v\left(I+G_{0} V\right) g=0 .
$$

Where we used the definition of $f$ and (50) in the last line. This completes the proof.

Lemma 5.2. Assume that $|V(x)| \lesssim\langle x\rangle^{-\beta}$ for some $\beta>2$, if $g \in L^{2}\left(\mathbb{R}^{n}\right)$ for $n \geq 5$ is a solution of $(-\Delta+V) g=0$ then $g \in L^{\infty}\left(\mathbb{R}^{n}\right)$.

Proof. Recall that if $(-\Delta+V) g=0$ is equivalent to $\left(I+G_{0} V\right) g=0$, so that

$$
g=-G_{0} V g
$$

Recall that $G_{0}(x, y)=c_{0}|x-y|^{2-n}$, so that we can write

$$
g=-G_{l o c} V g-G_{d i s t} V g,
$$

where

$$
G_{l o c}(x, y):=\frac{c_{0}}{|x-y|^{n-2}} \chi_{\left\{|x-y|<\epsilon_{n}\right\}}, \quad G_{d i s t}:=G_{0}-G_{l o c} .
$$

Here $\epsilon_{n}>0$ is a constant chosen so that

$$
\sup _{x \in \mathbb{R}^{n}} \int_{\mathbb{R}^{n}}\left|G_{l o c}(x, y) V(y)\right| d y<\frac{1}{2} .
$$

We can take $\epsilon_{n}<\left(c_{0}\|V\|_{\infty}^{2} \omega_{n-1}\right)^{-\frac{1}{2}}$ with $\omega_{n-1}$ the surface area of the unit ball in $\mathbb{R}^{n-1}$. Then,

$$
\left\|G_{\text {dist }} V g\right\|_{\infty} \leq\left\|G_{d i s t} V\right\|_{2}\|g\|_{2}=C\left\|_{g}\right\|_{2}
$$

since the $G_{d i s t}(x, y) V(y)$ is an $L^{2}$ function of $y$ uniformly in $x$. So that,

$$
\begin{aligned}
|g(x)| & =\left|\int_{\mathbb{R}^{n}}-\left(G_{l o c}(x, y) V(y)+G_{\text {dist }}(x, y) V(y)\right) g(y) d y\right| \\
& \leq\left(\int_{\mathbb{R}^{n}}\left|G_{l o c}(x, y) V(y)\right| d y\right)\|g\|_{\infty}+\left\|G_{d i s t} V\right\|_{2}\|g\|_{2}<\frac{1}{2}\|g\|_{\infty}+C\|g\|_{2} .
\end{aligned}
$$

Thus

$$
\|g\|_{\infty}<2 C\|g\|_{2}<\infty
$$

Lemma 5.3. The kernel of $S_{1} v G_{2} v S_{1}$ is trivial in $S_{1} L^{2}\left(\mathbb{R}^{n}\right)$ for $n \geq 5$. 
Proof. Take $f \in S_{1} L^{2}$ (that is such that $\left(U+v G_{0} v\right) f=0$ ) with $S_{1} v G_{2} v S_{1} f=0$. Then using (6) we have $G_{2}=\lim _{\lambda \rightarrow 0} \frac{R_{0}^{ \pm}\left(\lambda^{2}\right)-G_{0}}{\lambda^{2}}$.

$$
\begin{aligned}
0 & =\left\langle S_{1} v G_{2} v S_{1} f, f\right\rangle=\left\langle G_{2} v f, v f\right\rangle=\lim _{\lambda \rightarrow 0}\left\langle\left(\frac{R_{0}^{ \pm}\left(\lambda^{2}\right)-G_{0}}{\lambda^{2}}\right) v f, v f\right\rangle \\
& =\lim _{\lambda \rightarrow 0} \frac{1}{\lambda^{2}} \int\left(\left(|\xi|^{2}+\lambda^{2}\right)^{-1}-|\xi|^{2}\right)^{-1} \widehat{v f}(\xi) \widehat{\widehat{v f}}(\xi) d \xi \\
& =\lim _{\lambda \rightarrow 0} \int \frac{1}{|\xi|^{2}\left(|\xi|^{2}+\lambda^{2}\right)}|\widehat{v f}(\xi)|^{2} d \xi=\int \frac{|\widehat{v f}(\xi)|^{2}}{|\xi|^{4}} d \xi=\left\langle G_{0} v f, G_{0} v f\right\rangle .
\end{aligned}
$$

Where we used the monotone convergence theorem on the last equality. This implies that $\widehat{v f}=0$ and $v f=0$. Thus the kernel of $S v G_{2} v S$ is trivial.

Technically the above proof only applies in odd dimensions. It is easy to adapt the proof to even dimensions using the expansions given in the companion paper, [12] with the operator $G_{1}^{0}$ replacing $G_{2}$.

Corollary 5.4. For $f_{1}, f_{2} \in S_{1} L^{2}$ we have the identity

$$
\left\langle G_{2} v f_{1}, v f_{2}\right\rangle=\left\langle G_{0} v f_{1}, G_{0} v f_{2}\right\rangle .
$$

Lemma 5.5. The projection onto the eigenspace at zero is $G_{0} v S_{1}\left[S_{1} v G_{2} v S_{1}\right]^{-1} S_{1} v G_{0}$.

Proof. If $\operatorname{dim} S_{1} L^{2}=N<\infty$ (see [16]), then let $\phi_{j}, j=1,2, \ldots, N$ be an orthonormal basis for $S_{1} L^{2}\left(\mathbb{R}^{n}\right)$. Then

$$
\begin{aligned}
& 0=\left(U+v G_{0} v\right) \phi_{j}, \\
& 0=\left(I+w G_{0} v\right) \phi_{j}=\phi_{j}+w G_{0} v \phi_{j} .
\end{aligned}
$$

Write $\phi_{j}=w \psi_{j}$ for $1 \leq j \leq N$ with $\psi_{j}$ linearly independent. So that

$$
0=w \psi_{j}+w G_{0} v w \psi_{j}
$$

and so

$$
0=\psi_{j}+G_{0} V \psi_{j}
$$

So that for any $f \in L^{2}$ we have

$$
\begin{aligned}
S_{1} f & =\sum_{j=1}^{N}\left\langle f, \phi_{j}\right\rangle \phi_{j} \\
S_{1} v G_{0} f & =\sum_{j=1}^{N}\left\langle S_{1} v G_{0} f, \phi_{j}\right\rangle \phi_{j}=\sum_{j=1}^{N}\left\langle f, G_{0} v \phi_{j}\right\rangle \phi_{j}=-\sum_{j=1}^{N}\left\langle f, \psi_{j}\right\rangle \phi_{j}
\end{aligned}
$$


Let $A_{i j}$ be the matrix representation of $S_{1} v G_{2} v S_{1}$ with respect to $\left\{\phi_{j}\right\}_{j=1}^{N}$. That is,

$$
A_{i j}=\left\langle\phi_{i}, S_{1} v G_{2} v S_{1} \phi_{j}\right\rangle=\left\langle G_{0} v \phi_{i}, G_{0} v \phi_{j}\right\rangle=\left\langle G_{0} V \phi_{i}, G_{0} V \phi_{j}\right\rangle=\left\langle\psi_{i}, \psi_{j}\right\rangle .
$$

Denoting $P_{e}=G_{0} v S_{1}\left[S_{1} v G_{2} v S_{1}\right]^{-1} S_{1} v G_{0}$, for $f \in L^{2}$ we have

$$
\begin{aligned}
P_{e} f & =G_{0} v S_{1}\left[S_{1} v G_{2} v S_{1}\right]^{-1} S_{1} v G_{0} f=G_{0} v S_{1}\left[S_{1} v G_{2} v S_{1}\right]^{-1}\left(-\sum_{j=1}^{N}\left\langle f, \psi_{j}\right\rangle \phi_{j}\right) \\
& =-\sum_{j=1}^{N} G_{0} v S_{1}\left[S_{1} v G_{2} v S_{1}\right]^{-1} \phi_{j}\left\langle f, \psi_{j}\right\rangle=\sum_{i, j=1}^{N} G_{0} v S_{1}\left(A_{i j}^{-1}\right) \phi_{i}\left\langle f, \psi_{j}\right\rangle \\
& =-\sum_{i, j=1}^{N} G_{0} v \phi_{i}\left(A_{i j}^{-1}\right)\left\langle f, \psi_{j}\right\rangle=\sum_{i, j=1}^{N}\left(A_{i j}^{-1}\right) \psi_{i}\left\langle f, \psi_{j}\right\rangle .
\end{aligned}
$$

For $f=\psi_{k}$ we have

$$
P_{e} \psi_{k}=\sum_{i, j=1}^{N}\left(A_{i j}^{-1}\right) \psi_{i}\left\langle\psi_{k}, \psi_{j}\right\rangle=\sum_{i, j=1}^{N}\left(A_{i j}^{-1}\right)\left(A_{j k}\right) \psi_{i}=\psi_{k}
$$

Thus, we have that the range of $P_{e}$ is the span of $\left\{\psi_{j}\right\}_{j=1}^{N}$ and is the identity on the range of $P_{e}$. Since $P_{e}$ is self-adjoint, we are done.

Defining $P_{e}$ to be the projection onto the zero energy eigenspace, to match the notation of the previous sections we have

$$
P_{e}=G_{0} v D_{1} v G_{0}
$$

We also have the following corollary.

Corollary 5.6. $P_{e}$ is bounded operator from $L^{1}$ to $L^{\infty}$.

Proof. Take $f \in L^{1}$, then

$$
\left|P_{e} f(x)\right|=\left|\sum_{j=1}^{N}\left\langle\psi_{j}, f\right\rangle \psi_{j}\right| \leq \sum_{j=1}^{N}\left|\left\langle\psi_{j}, f\right\rangle\right|\left|\psi_{j}\right| \leq \sum_{j=1}^{N}\left\|\psi_{j}\right\|_{\infty}^{2}\|f\|_{1}<\infty
$$

by Lemma [5.2, $\psi_{j} \in L^{\infty}$. 
5.1. Prospective examples of $V(x)$. Here we construct examples of potentials $V$ for which $H=-\Delta+V$ has a zero-energy eigenvalue whose eigenfunction $\psi$ satisfies $\int V \psi=0$ and $\int x V \psi=0$. This shows that the hypotheses in Theorem 1.2 and Corollary 1.3 can be realized.

Any solution of the equation $(-\Delta+V) \psi=0$ satisfies the functional relation $-\psi=$ $(-\Delta)^{-1}(V \psi)$, where $(-\Delta)^{-1}$ is convolution against the Green's function $C_{n}|x|^{2-n}$. If $V$ is compactly supported, then $\psi$ is a harmonic function outside the support of $V$ and decays at the rate $|x|^{2-n}$ unless additional cancellation takes place inside the convolution integral. Choose any (nonempty) finite collection of points $x_{i} \in \mathbb{R}^{n}$, and weights $\mu_{i} \in \mathbb{R}$ so that the signed measure $\sum_{i} \mu_{i} \delta_{x_{i}}$ has vanishing moments up to $k^{\text {th }}$ order. Then let

$$
F_{k}(x):=\left|\Delta^{-1}\left(\sum_{i} \mu_{i} \delta_{x_{i}}\right)\right|=-C_{n} \sum_{i} \mu_{i}\left|x-x_{i}\right|^{2-n} .
$$

When $|x|>2 \max \left|x_{i}\right|$, we can expand each $\left|x-x_{i}\right|^{2-n}$ as a Taylor series centered at $x$, that is $\left|x-x_{i}\right|^{2-n}=P_{k}\left(x_{i}\right)+O\left(|x|^{1-n-k}\left|x_{i}\right|^{k+1}\right)$. The vanishing moment assumption ensures that $\sum_{i} \mu_{i} P_{k}\left(x_{i}\right)=0$, which leaves $F_{k}(x)=\sum_{i} \mu_{i} O\left(|x|^{1-n-k}\left|x_{i}\right|^{k+1}\right) \lesssim|x|^{1-n-k}$.

Each $x_{i}$ has a neighborhood $\Omega_{i}$ where $F_{k}(x) \sim \mu_{i}\left|x-x_{i}\right|^{2-n}$ and is therefore nonzero. Now let $\psi_{k}(x)$ be any function that agrees with $F_{k}(x)$ outside of the union of $\Omega_{i}$ and is a nonvanishing $C^{2}$ continuation inside. Then $\Delta \psi_{k}$ is continuous with compact support inside $\cup_{i} \bar{\Omega}_{i}$. Finally,

$$
V(x)=\frac{\Delta \psi_{k}(x)}{\psi_{k}(x)}
$$

belongs to $C_{c}\left(\mathbb{R}^{n}\right)$ and the Schrödinger operator $H=-\Delta+V$ has $\psi_{k}$ as a rapidly decaying eigenfunction at $\lambda=0$. Specifically, $\left|\psi_{k}(x)\right| \lesssim|x|^{1-n-k}$ for large $|x|$.

The conditions of Theorem [1.2, part 3 are satisfied for any potential constructed in this manner with $k \geq 1$, provided all other eigenfunctions (if any exist) also decay at the rate $|x|^{-n}$ or faster. The following argument adapted from [11] suggests that typically the eigenspace is in fact one-dimensional.

Starting from a fixed choice of $\psi_{k}$ it is possible to construct a larger family of complex potentials of the form $\psi_{z}(x)=\psi_{k}(x) e^{z \eta(x)}$, where $\eta \in C_{c}^{\infty}\left(\cup_{i} \Omega_{i}\right)$ and $z$ varies over $\mathbb{C}$. The resulting potentials $V_{z}(x)$ have complex-analytic dependence on $z$. The analytic Fredholm theorem applied to $\left(I+(-\Delta)^{-1} V_{z}\right)$ indicates that the dimension of the nullspace for $-\Delta+V_{z}$ should be constant for generic $z$ with only a discrete set of exceptions where it is larger. 
If the nullspace of $H_{0}:=-\Delta+V_{0}$ is one-dimensional, we are done. Supose the nullspace is two-dimensional with a second eigenfunction $\phi$. As constructed, the formula for $V_{z}$ is

$$
V_{z}=\frac{\Delta\left(e^{z \eta} \psi_{k}\right)}{e^{z \eta} \psi_{k}}=V_{0}-\frac{H_{0}\left(\eta \psi_{k}\right)}{\psi_{k}} z+\frac{|\nabla \eta|^{2}}{\psi_{k}} z^{2}
$$

In particular, $\left.\frac{d}{d z} V_{z}\right|_{z=0}=-\frac{H_{0}\left(\eta \psi_{k}\right)}{\psi_{k}}$.

Then for small values of $z$,

$$
\begin{aligned}
\left\langle\left(-\Delta+V_{z}\right) \phi, \phi\right\rangle & =\left\langle\left(-\Delta+V_{0}\right) \phi, \phi\right\rangle-\left\langle\left(\frac{H_{0}\left(\eta \psi_{k}\right)}{\psi_{k}}\right) \phi, \phi\right\rangle z+O\left(z^{2}\right) \\
& =-\left\langle H_{0}\left(\eta \psi_{k}\right), \frac{\phi^{2}}{\psi_{k}}\right\rangle z+O\left(z^{2}\right) .
\end{aligned}
$$

If the leading-order term is nonzero, then the repeated eigenvalue when $z=0$ will split for all other nearby values of $z$. However, since $H_{0}$ is self-adjoint, we can rewrite the inner product as

$$
\left\langle H_{0}\left(\eta \psi_{k}\right), \frac{\phi^{2}}{\psi_{k}}\right\rangle=\left\langle\eta, \psi_{k} H_{0}\left(\frac{\phi^{2}}{\psi_{k}}\right)\right\rangle .
$$

Since $\psi_{k}$ and $\phi$ are linearly independent, $\frac{\phi^{2}}{\psi_{k}}$ is linearly independent from both of them, thus the function on the right side of the inner product is nonzero. Any choice of $\eta$ which is not orthogonal to this suffices for constructing potentials $V_{z}$ that have a one-dimensional eigenspace at zero (with eigenvector $e^{z \eta} \psi_{k}$ ).

Similar eigenvalue-splitting arguments hold if the nullspace of $H_{0}$ is larger, provided a sufficient collection of functions $H_{0}\left(\frac{\phi_{i} \phi_{j}}{\psi_{k}}\right)$ are nonzero and linearly independent.

\section{Integral Estimates}

The proof of Lemma 2.2 hinges on the following estimate.

Lemma 6.1. Fix $u_{1}, u_{2} \in \mathbb{R}^{n}$ and let $0 \leq k, \ell<n, \beta>0, k+\ell+\beta \geq n, k+\ell \neq n$. We have

$$
\int_{\mathbb{R}^{n}} \frac{\langle z\rangle^{-\beta-}}{\left|z-u_{1}\right|^{k}\left|z-u_{2}\right|^{\ell}} d z \lesssim \begin{cases}\left(\frac{1}{\left|u_{1}-u_{2}\right|}\right)^{\max (0, k+\ell-n)} & \left|u_{1}-u_{2}\right| \leq 1 \\ \left(\frac{1}{\left|u_{1}-u_{2}\right|}\right)^{\min (k, \ell, k+\ell+\beta-n)} & \left|u_{1}-u_{2}\right|>1\end{cases}
$$

More precisely, noting that $\max (0, k+\ell-n) \leq \min (k, \ell, k+\ell+\beta-n)$, we use the simple corollary,

Corollary 6.2. Fix $u_{1}, u_{2} \in \mathbb{R}^{n}$ and let $0 \leq k, \ell<n, \beta>0, k+\ell+\beta \geq n, k+\ell \neq n$. We have

$$
\int_{\mathbb{R}^{n}} \frac{\langle z\rangle^{-\beta-}}{\left|z-u_{1}\right|^{k}\left|z-u_{2}\right|^{\ell}} d z \lesssim\left(\frac{1}{\left|u_{1}-u_{2}\right|}\right)^{\max (0, k+\ell-n)}
$$


and

$$
\int_{\mathbb{R}^{n}} \frac{\langle z\rangle^{-\beta-}}{\left|z-u_{1}\right|^{k}\left|z-u_{2}\right|^{\ell}} d z \lesssim\left(\frac{1}{\left|u_{1}-u_{2}\right|}\right)^{\min (k, \ell, k+\ell+\beta-n)} .
$$

Proof of Lemma 2.2. We note the bound

$$
\left|R_{0}^{ \pm}\left(\lambda^{2}\right)(x, y)\right| \lesssim \frac{1}{|x-y|^{n-2}}+\frac{\lambda^{\frac{n-3}{2}}}{|x-y|^{\frac{n-1}{2}}},
$$

which follows from (5). To control local singularities, it is only necessary to bound integrals of the form

$$
\iint_{\mathbb{R}^{k n}} \frac{1}{\left|x-z_{1}\right|^{n-2}} \prod_{j=1}^{n} \frac{\left\langle z_{j}\right\rangle^{-\beta-}}{\left|z_{j}-z_{j+1}\right|^{n-2}} d \vec{z},
$$

where $z_{n+1}=y$ and $d \vec{z}=d z_{1} d z_{2} \ldots d z_{n}$. For this integral we iterate the first bound in Corollary 6.2. Since $\ell=n-2$ at each step we have that $k+\ell-n=k-2$, that is we improve the local singularity by two powers after each integral. So that, after $m$ integrals of this form the singularity is $\left|x-z_{m}\right|^{n-2(m+1)}$, so that we need $n-2(m+1) \leq \frac{n-1}{2}$ to ensure the iterated integral is locally $L^{2}$. We take $\kappa=\frac{n-1}{4}$ or $\frac{n-3}{4}$ whichever is an integer. At the final step if $n \equiv 1 \bmod 4$ we have,

$$
\int_{\mathbb{R}^{n}} \frac{\left\langle z_{\kappa}\right\rangle^{-\beta-}}{\left|x-z_{\kappa}\right|^{\frac{n+1}{2}}\left|z_{\kappa}-y\right|^{n-2}} d z_{\kappa} \lesssim \begin{cases}|x-y|^{\frac{3-n}{2}} & |x-y| \leq 1 \\ |x-y|^{-\frac{n+1}{2}} & |x-y| \geq 1\end{cases}
$$

which is in $L_{y}^{2}$ uniformly in $x$. On the other hand, if $n \equiv 3 \bmod 4$ we have

$$
\int_{\mathbb{R}^{n}} \frac{\left\langle z_{\kappa}\right\rangle^{-\beta-}}{\left|x-z_{\kappa}\right|^{\frac{n+3}{2}}\left|z_{\kappa}-y\right|^{n-2}} d z_{\kappa} \lesssim \begin{cases}|x-y|^{\frac{1-n}{2}} & |x-y| \leq 1 \\ |x-y|^{-\frac{n+3}{2}} & |x-y| \geq 1\end{cases}
$$

which is also in $L_{y}^{2}$ uniformly in $x$.

To determine the weight needed, we need only control integrals of the form

$$
\iint_{\mathbb{R}^{k n}} \frac{1}{\left|x-z_{1}\right|^{\frac{n-1}{2}}} \prod_{j=1}^{n} \frac{\left\langle z_{j}\right\rangle^{-\beta-}}{\left|z_{j}-z_{j+1}\right|^{\frac{n-1}{2}}} d \vec{z} .
$$

Here we use the second bound in Corollary 6.2 for when $k, \ell=\frac{n-1}{2}$ we have

$$
\int_{\mathbb{R}^{n}} \frac{\langle z\rangle^{-\beta-}}{\left|z-u_{1}\right|^{k}\left|z-u_{2}\right|^{\ell}} d z \lesssim\left(\frac{1}{\left|u_{1}-u_{2}\right|}\right)^{\min \left(\frac{n-1}{2}, \beta-1\right)}
$$

If $\beta>\frac{n+1}{2}$, then $\min \left(\frac{n-1}{2}, \beta-1\right)=\frac{n-1}{2}$ and we can iterate this bound to see that

$$
|(\underline{53})| \lesssim \frac{1}{|x-y|^{\frac{n-1}{2}}} \in L_{y}^{2,-\frac{1}{2}-}\left(\mathbb{R}^{n}\right)
$$

uniformly in $x$. Any other terms that appear in the product of free resolvents can be seen to be bounded by a sum of integrals of the form we considered here. 
Lemma 6.3. If $k \in \mathbb{N}_{0}$, we have the bound

$$
\left|\int_{0}^{\infty} e^{i t \lambda^{2}} \chi(\lambda) \lambda^{k} d \lambda\right| \lesssim|t|^{-\frac{k+1}{2}}
$$

Proof. We employ the identity $\frac{d}{d \lambda} e^{i t \lambda^{2}}=2 i t \lambda e^{i t \lambda^{2}}$ to integrate by parts against the imaginary Gaussian. Formally, upon integrating by parts $m$ times we have

$$
\left.\left|\int_{0}^{\infty} e^{i t \lambda^{2}} \chi(\lambda) \lambda^{k} d \lambda\right| \lesssim \sum_{j=0}^{m-1} \frac{\chi(\lambda) \lambda^{k-1-2 j}}{|t|^{j+1}}\right|_{\lambda=0} ^{\infty}+\frac{1}{|t|^{m}}\left|\int_{0}^{\infty} e^{i t \lambda^{2}} \chi(\lambda) \lambda^{k-2 m} d \lambda\right|+O\left(|t|^{-\ell}\right) .
$$

The first term collects the boundary terms in the integration by parts, while the final $O\left(|t|^{-\ell}\right)$ term for arbitrary $\ell \geq 0$ is obtained from any term where the derivative acts on the cut-off function $\chi(\lambda)$. This follows since $\chi^{\prime}(\lambda)$ is supported on the annulus $\lambda \approx 1$ and one can integrate by parts arbitrarily many times with no boundary terms or convergence issues.

If $k$ is odd, we select $m=\frac{k-1}{2}$. The boundary terms are all zero since $k-1-2 j \geq 2$. Meanwhile, one additional integration by parts yields

$$
\frac{1}{|t|^{\frac{k-1}{2}}}\left|\int_{0}^{\infty} e^{i t \lambda^{2}} \lambda \chi(\lambda) d \lambda\right| \lesssim \frac{1}{|t|^{\frac{k+1}{2}}}+\frac{1}{|t|^{\frac{k+1}{2}}} \int_{0}^{\infty} e^{i t \lambda^{2}} \chi^{\prime}(\lambda) d \lambda \lesssim|t|^{-\frac{k+1}{2}} .
$$

On the other hand, if $k$ is even, we select $m=\frac{k}{2}$ and note that all the boundary terms are zero since $k-1-2 j \geq 1$. The integral bound

$$
\frac{1}{|t|^{\frac{k}{2}}}\left|\int_{0}^{\infty} e^{i t \lambda^{2}} \chi(\lambda) d \lambda\right| \lesssim \frac{1}{|t|^{\frac{k+1}{2}}}
$$

follows from Parseval's Identity since

$$
\left\|\widehat{e^{i t(\cdot)^{2}}}\right\|_{\infty} \lesssim|t|^{-\frac{1}{2}}, \quad \text { and } \quad\left\|\chi^{\vee}(\cdot)\right\|_{1} \lesssim 1
$$

The above calculations do not immediately apply to bounding integrals of the form

$$
\int_{0}^{\infty} e^{i t \lambda^{2}} f(\lambda) d \lambda
$$

when $f$ and its derivatives are bounded by powers of $\lambda$. Accordingly, we have the following oscillatory integral bound. 
Lemma 6.4. For a fixed $\alpha>-1$, let $f(\lambda)=\widetilde{O}_{k+1}\left(\lambda^{\alpha}\right)$ be supported on the interval $\left[0, \lambda_{1}\right]$ for some $0<\lambda_{1} \lesssim 1$. Then, if $k$ satisfies $-1<\alpha-2 k<1$ we have

$$
\left|\int_{0}^{\infty} e^{i t \lambda^{2}} f(\lambda) d \lambda\right| \lesssim|t|^{-\frac{\alpha+1}{2}}
$$

Proof. As in the proof of Lemma 6.3 we can integrate by parts $k$ times without having boundary terms since $\alpha-2 k+2>1$. At this point, we need only bound

$$
\frac{1}{|t|^{k}} \int_{0}^{\infty} e^{i t \lambda^{2}} g(\lambda) d \lambda
$$

Here $g(\lambda)=\widetilde{O}_{1}\left(\lambda^{\alpha-2 k}\right)$ is again supported on $\left[0, \lambda_{1}\right]$. By the definition of the integer $k$ we have $-1<\alpha-2 k<1$ so that further integration by parts is not possible. Without loss of generality we take $t>0$ and we break the integral into two parts,

$$
|(\underline{54})| \lesssim \frac{1}{|t|^{k}} \int_{0}^{t^{-\frac{1}{2}}}|g(\lambda)| d \lambda+\frac{1}{|t|^{k}}\left|\int_{t^{-\frac{1}{2}}}^{\infty} e^{i t \lambda^{2}} g(\lambda) d \lambda\right| .
$$

The bound for the first integral follows from integration,

$$
\int_{0}^{t^{-\frac{1}{2}}}|g(\lambda)| d \lambda \lesssim \int_{0}^{t^{-\frac{1}{2}}} \lambda^{\alpha-2 k} d \lambda \lesssim|t|^{-\frac{\alpha}{2}+k-\frac{1}{2}}
$$

Since $-1<\alpha-2 k$ this is integrable at zero. For the second integral, we integrate by parts again. As $\alpha-2 k<1$ there is no a boundary term at infinity, but we do have one at $t^{-\frac{1}{2}}$. Thus we see

$$
\left.\int_{t^{-\frac{1}{2}}}^{\infty} e^{i t \lambda^{2}} g(\lambda) d \lambda \lesssim \frac{g(\lambda)}{\lambda t}\right|_{\lambda=t^{-\frac{1}{2}}}+\frac{1}{t} \int_{t^{-\frac{1}{2}}}^{\infty} \lambda^{\alpha-2 k-2} d \lambda \lesssim|t|^{-\frac{\alpha}{2}+k-\frac{1}{2}}
$$

The final integrand is integrable as $\alpha-2 k-2<-1$.

We need the following lemma which is a modification of stationary phase.

Lemma 6.5. We have the bound

$$
\left|\int_{t^{-\frac{1}{2}}}^{1} e^{i t\left(\lambda-\lambda_{0}\right)^{2}} F(\lambda, x, y) \chi(\lambda) d \lambda\right| \lesssim t^{-\frac{1}{2}} \sup _{\lambda}|F(\lambda, x, y)|+t^{-\frac{3}{4}}\left[\int_{t^{-\frac{1}{2}}}^{1}\left|\frac{\partial F}{\partial \lambda}(\lambda, x, y)\right|^{2} d \lambda\right]^{\frac{1}{2}} .
$$

Proof. Assume that $t>0$, the proof for $t<0$ proceeds identically. We note that in the case when $\lambda_{0} \ll t^{-\frac{1}{2}}$ or $\lambda_{0} \gg 1$ we can integrate by parts safely against $e^{i t\left(\lambda-\lambda_{0}\right)^{2}}$ since $\left|\lambda-\lambda_{0}\right| \gtrsim \lambda$. Then, we have

$$
\left|\int_{t^{-\frac{1}{2}}}^{1} e^{i t\left(\lambda-\lambda_{0}\right)^{2}} F(\lambda, x, y) \chi(\lambda) d \lambda\right|
$$




$$
\left.\lesssim \frac{F(\lambda, x, y) \chi(\lambda)}{\lambda t}\right|_{t^{-\frac{1}{2}}} ^{1}+\frac{1}{t} \int_{t^{-\frac{1}{2}}}^{1}\left|\frac{F(\lambda, x, y)}{\lambda^{2}}\right|+\left|\frac{\partial_{\lambda} F(\lambda, x, y)}{\lambda}\right| d \lambda
$$

The first two terms can be seen to be bounded by $t^{-\frac{1}{2}} \sup _{\lambda}|F(\lambda, x, y)|$. The second term is bounded by applying Cauchy-Schwartz.

On the other hand, if $t^{-\frac{1}{2}} \lesssim \lambda_{0} \lesssim 1$ we instead consider

$$
e^{i t \lambda_{0}^{2}} \int_{t^{-\frac{1}{2}}}^{1} e^{i t \lambda^{2}} e^{-2 i t \lambda_{0} \lambda} F(\lambda, x, y) d \lambda
$$

Here we can integrate by parts once, and ignore the constant $e^{i t \lambda_{0}^{2}}$ to bound

$$
t^{-\frac{1}{2}} \sup _{\lambda}|F(\lambda, x, y)|+\frac{1}{t} \int_{t^{-\frac{1}{2}}}^{1}|F(\lambda, x, y)|\left(\lambda_{0} \lambda^{-1}+\lambda^{-2}\right)+\frac{\left|\partial_{\lambda} F(\lambda, x, y)\right|}{\lambda} d \lambda
$$

Since $\lambda_{0} \lesssim 1$, we see that

$$
\begin{gathered}
\frac{1}{t} \int_{t^{-\frac{1}{2}}}^{1}|F(\lambda, x, y)|\left(\lambda_{0} \lambda^{-1}+\lambda^{-2}\right) d \lambda \lesssim \frac{\sup _{\lambda}|F(\lambda, x, y)|}{t} \int_{t^{-\frac{1}{2}}}^{1}\left(\frac{\lambda_{0}}{\lambda}+\frac{1}{\lambda^{2}}\right) d \lambda \\
\lesssim \frac{\sup _{\lambda}|F(\lambda, x, y)|}{t} \int_{t^{-\frac{1}{2}}}^{1}\left(t^{\frac{1}{2}}+\frac{1}{\lambda^{2}}\right) d \lambda \lesssim \frac{\sup _{\lambda}|F(\lambda, x, y)|}{t^{\frac{1}{2}}}
\end{gathered}
$$

The final term is bounded by Cauchy-Schwartz,

$$
\begin{aligned}
\frac{1}{t} \int_{t^{-\frac{1}{2}}}^{1} \frac{\left|\partial_{\lambda} F(\lambda, x, y)\right|}{\lambda} d \lambda & \lesssim \frac{1}{t}\left(\int_{t^{-\frac{1}{2}}}^{\infty} \lambda^{-2} d \lambda\right)^{\frac{1}{2}}\left(\int_{t^{-\frac{1}{2}}}^{1}\left|\partial_{\lambda} F(\lambda, x, y)\right| d \lambda\right)^{\frac{1}{2}} \\
& \lesssim|t|^{-\frac{3}{4}}\left(\int_{t^{-\frac{1}{2}}}^{1}\left|\partial_{\lambda} F(\lambda, x, y)\right| d \lambda\right)^{\frac{1}{2}}
\end{aligned}
$$

Finally, we remark that the oscillatory bounds in Lemmas 6.3 and 6.5 hold if the cut-off function $\chi(\lambda)$ is supported on $\left[0, \lambda_{1}\right]$ for any finite $\lambda_{1}$.

\section{REFERENCES}

[1] Abramowitz, M. and I. A. Stegun. Handbook of mathematical functions with formulas, graphs, and mathematical tables. National Bureau of Standards Applied Mathematics Series, 55. For sale by the Superintendent of Documents, U.S. Government Printing Office, Washington, D.C. 1964

[2] Agmon, S. Spectral properties of Schrödinger operators and scattering theory. Ann. Scuola Norm. Sup. Pisa Cl. Sci. (4) 2 (1975), no. 2, 151-218.

[3] Beceanu, M. and Goldberg, M. Schrödinger dispersive estimates for a scaling-critical class of potentials. Comm. Math. Phys. 314 (2012), no. 2, 471-481.

[4] Cardosa, F., Cuevas, C., and Vodev, G. Dispersive estimates for the Schrödinger equation in dimensions four and five. Asymptot. Anal. 62 (2009), no. 3-4, 125-145.

[5] Erdoğan, M. B., Goldberg, M. J., and Green, W. R. Dispersive estimates for four dimensional Schrödinger and wave equations with obstructions at zero energy. Comm. PDE. 39, no. 10, 1936-1964. 
[6] Erdoğan, M. B. and Green, W. R. Dispersive estimates for the Schrodinger equation for $C^{\frac{n-3}{2}}$ potentials in odd dimensions. Int. Math. Res. Notices 2010:13, 2532-2565.

[7] Erdoğan, M. B. and Green, W. R. Dispersive estimates for Schrödinger operators in dimension two with obstructions at zero energy. Trans. Amer. Math. Soc. 365 (2013), 6403-6440.

[8] Erdoğan, M. B., and Schlag, W. Dispersive estimates for Schrödinger operators in the presence of a resonance and/or eigenvalue at zero energy in dimension three: II. J. Anal. Math. 99 (2006), 199-248.

[9] Erdoğan, M. B. and Schlag W. Dispersive estimates for Schrödinger operators in the presence of a resonance and/or an eigenvalue at zero energy in dimension three: I. Dynamics of PDE 1 (2004), 359379.

[10] Finco, D. and Yajima, K. The $L^{p}$ boundedness of wave operators for Schrödinger operators with threshold singularities II. Even dimensional case. J. Math. Sci. Univ. Tokyo 13 (2006), no. 3, 277-346.

[11] Goldberg, M. A Dispersive Bound for Three-Dimensional Schrödinger Operators with Zero Energy Eigenvalues. Comm. PDE 35, no. 9 (2010), 1610-1634.

[12] Goldberg, M. and Green, W. Dispersive Estimates for higher dimensional Schrödinger Operators with threshold eigenvalues II: The even dimensional case. To appear in J. Spectr. Theory, 2014.

[13] Goldberg, M. and Schlag, W. Dispersive estimates for Schrödinger operators in dimensions one and three. Comm. Math. Phys. vol. 251, no. 1 (2004), 157-178.

[14] Goldberg, M. and Visan, M. A Counterexample to Dispersive Estimates. Comm. Math. Phys. 266 (2006), no. $1,211-238$.

[15] Green, W. Dispersive estimates for matrix and scalar Schrödinger operators in dimension five. Illinois J. Math. Volume 56, Number 2 (2012), 307-341.

[16] Jensen, A. Spectral properties of Schrödinger operators and time-decay of the wave functions results in $L^{2}\left(R^{m}\right), m \geq 5$. Duke Math. J. 47 (1980), no. 1, 57-80.

[17] Jensen, A. Spectral properties of Schrödinger operators and time-decay of the wave functions. Results in $L^{2}\left(R^{4}\right)$. J. Math. Anal. Appl. 101 (1984), no. 2, 397-422.

[18] Jensen, A. and Kato, T. Spectral properties of Schrödinger operators and time-decay of the wave functions. Duke Math. J. 46 (1979), no. 3, 583-611.

[19] Jensen, A. and Nenciu, G. A unified approach to resolvent expansions at thresholds. Rev. Mat. Phys. 13, no. 6 (2001), 717-754.

[20] Jensen, A., and Yajima, K. On $L^{p}$ boundedness of wave operators for 4-dimensional Schrödinger operators with threshold singularities. Proc. Lond. Math. Soc. (3) 96 (2008), no. 1, 136-162.

[21] Journé, J.-L., Soffer, and A., Sogge, C. D. Decay estimates for Schrödinger operators. Comm. Pure Appl. Math. 44 (1991), no. 5, 573-604.

[22] Murata, M. Asymptotic expansions in time for solutions of Schrödinger-type equations. J. Funct. Anal. 49 (1) (1982), 10-56.

[23] Rauch, J. Local decay of scattering solutions to Schrödinger's equation. Comm. Math. Phys. 61 (1978), no. 2, 149-168.

[24] Reed, M. and Simon, B. Methods of Modern Mathematical Physics I: Functional Analysis, IV: Analysis of Operators, Academic Press, New York, NY, 1972. 
[25] Rodnianski, I. and Schlag, W. Time decay for solutions of Schrödinger equations with rough and timedependent potentials. Invent. Math. 155 (2004), no. 3, 451-513.

[26] Schlag, W. Dispersive estimates for Schrödinger operators in dimension two. Comm. Math. Phys. 257 (2005), no. 1, 87-117.

[27] Schlag, W. Dispersive estimates for Schrödinger operators: a survey. Mathematical aspects of nonlinear dispersive equations, 255-285, Ann. of Math. Stud. 163, Princeton Univ. Press, Princeton, NJ, 2007.

[28] Weder, R. $L^{p}-L^{p^{\prime}}$ estimates for the Schrödinger equation on the line and inverse scattering for the nonlinear Schrödinger equation with a potential. J. Funct. Anal. 170 (2000), no. 1, 37-68.

[29] Yajima, K. Dispersive estimate for Schrödinger equations with threshold resonance and eigenvalue. Comm. Math. Phys. 259 (2005), 475-509.

[30] Yajima, K. The $L^{p}$ Boundedness of wave operators for Schrödinger operators with threshold singularities I. The odd dimensional case. J. Math. Sci. Univ. Tokyo 13 (2006), 43-94.

Department of Mathematics, University of Cincinnati, Cincinnati, OH 45221-0025

E-mail address: Michael.Goldberg@uc.edu

Department of Mathematics, Rose-Hulman Institute of Technology, Terre Haute, IN 47803 U.S.A.

E-mail address: green@rose-hulman.edu 